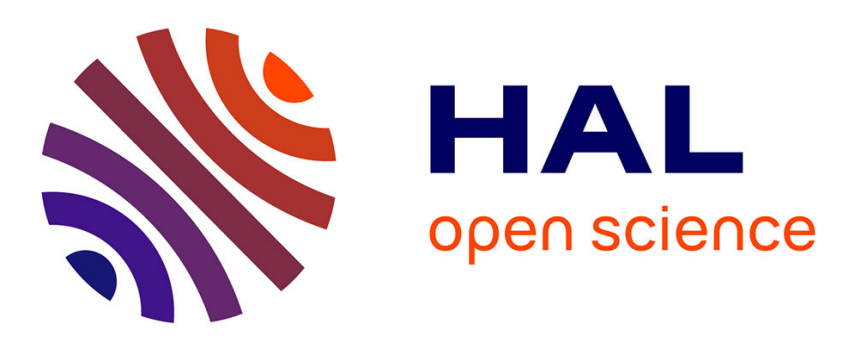

\title{
Interaction d'un faisceau laser avec une cible métallique - Première partie Étude de l'échauffement et de la vaporisation de la cible
}

\author{
J.-L. Dumas
}

\section{To cite this version:}

J.-L. Dumas. Interaction d'un faisceau laser avec une cible métallique - Première partie Étude de l'échauffement et de la vaporisation de la cible. Revue de Physique Appliquée, 1970, 5 (6), pp.795809. 10.1051/rphysap:0197000506079500 . jpa-00243457

\section{HAL Id: jpa-00243457 https://hal.science/jpa-00243457}

Submitted on 1 Jan 1970

HAL is a multi-disciplinary open access archive for the deposit and dissemination of scientific research documents, whether they are published or not. The documents may come from teaching and research institutions in France or abroad, or from public or private research centers.
L'archive ouverte pluridisciplinaire HAL, est destinée au dépôt et à la diffusion de documents scientifiques de niveau recherche, publiés ou non, émanant des établissements d'enseignement et de recherche français ou étrangers, des laboratoires publics ou privés. 


\title{
REVUE DE PHYSIQUE APPLIQUÉE
}

Supplément au « Journal de Physique *

\section{INTERACTION D'UN FAISCEAU LASER AVEC UNE CIBLE MÉTALLIQUE}

\author{
Première partie \\ ÉTUDE DE L'ÉCHAUFFEMENT \\ ET DE LA VAPORISATION DE LA CIBLE
}

\author{
par J.-L. DUMAS \\ Section d'Etudes et d'Analyses Physico-Chimiques, \\ Laboratoire de Spectrométrie de Masse, Centre d'Etudes Nucléaires de Grenoble
}

(Reçu le 25 février 1970, révisé le 22 mai 1970)

\begin{abstract}
Résumé. - Les dégâts provoqués sur une cible métallique, sous vide, par focalisation d'un faisceau lumineux issu d'un laser à rubis, sont fonction de l'énergie et de la durée d'impulsion lumineuse (dans le domaine de $2 \times 10^{-7} \mathrm{~s}$ à $2 \times 10^{-6} \mathrm{~s}$ et de 1 à $300 \mathrm{~mJ}$ ).

1. Lorsque l'énergie est insuffisante pour vaporiser les couches traversées, la propagation du front thermique n'a que des effets d'échauffement se traduisant par une modification de l'état de surface et la désorption des contaminants superficiels.

2. Lorsque l'énergie atteint une valeur seuil, 0,01 Joule délivré en $0,8 \mu$ s (soit environ $10^{7} \mathrm{~W} / \mathrm{cm}^{2}$ ), un cratère est formé, la pénétration du front thermique s'accompagnant alors de la vaporisation du matériau.

3. Les dimensions de ce cratère (profondeur, diamètre) augmentent avec l'énergie lumineuse, mais en tendant vers des valeurs limites pour des énergies de dix à cent fois supérieures au seuil.

On peut rendre compte de ce phénomène de saturation en considérant les vitesses relatives de pénétration de la chaleur et de propagation du front de vaporisation dans le matériau. Le modèle proposé permet de calculer la quantité de matière vaporisée (de l'ordre du microgramme) en fonction des paramètres de l'impulsion lumineuse et des propriétés physiques de la cible.
\end{abstract}

4. Au-delà du seuil, si la profondeur du cratère reste sensiblement constante, par contre la projection du matériau est plus violente.

Abstract. - The damages produced on a metallic target, under vacuum, by radiation from a focused ruby laser beam are a function of the energy and duration of the luminous pulse (approximately 1 to $300 \mathrm{~mJ}$ and 0.2 to $2 \mu \mathrm{s}$ ).

1. When the energy is insufficient to vaporize the layers traversed by the thermal front, its propagation has only heating effects shown by a surface change of state and desorption of superficial contaminants.

2. When the energy reaches a threshold, 0.01 joule delivered in $0.8 \mu \mathrm{s}$ (about $10^{7} \mathrm{~W} / \mathrm{cm}^{2}$ ), a crater is formed ; penetration of the thermal front then accompanies the vaporization of the material.

3. The dimensions of this crater (depth, diameter) increase with luminous energy, but approach limited values at energies ten to one-hundred times above the threshold.

One can account for this saturation phenomenon by considering the relative rates of heat penetration and of propagation of the vaporization front into the material. The proposed model allows calculation of the quantity of material vaporized (approximately a microgram) as a function of the luminous pulse parameters and physical properties of the target.

4. Beyond the threshold, if the crater depth remains reasonably constant, the ejection of material is more violent.

I. Introduction. - Le faisceau de lumière cohérente émis par un laser peut être facilement concentré sur une très petite surface; lorsque la puissance de la source lumineuse est suffisante, l'interaction se traduit par la formation d'un cratère dans la cible, accompagnée de l'émission de particules neutres et chargées.
Dès 1963 fut entreprise la réalisation de spectromètres de masse $[1,2]$ pour l'analyse localisée de surfaces solides à partir des ions produits directement $[3,4$, 5, 6].

Notre étude porte sur les deux aspects de l'interaction du laser avec la matière : dégâts sur la cible, 
d'une part, et production de particules chargées d'autre part.

Dans cette première partie nous avons étudié expérimentalement et théoriquement l'influence des paramètres lumineux (énergie et durée d'impulsion) sur la nature des dégâts et la quantité de matière vaporisée, en tenant compte des propriétés physiques de la cible.

II. Dispositif et méthode expérimentale. - Le dispositif expérimental est représenté sur la figure 1 .

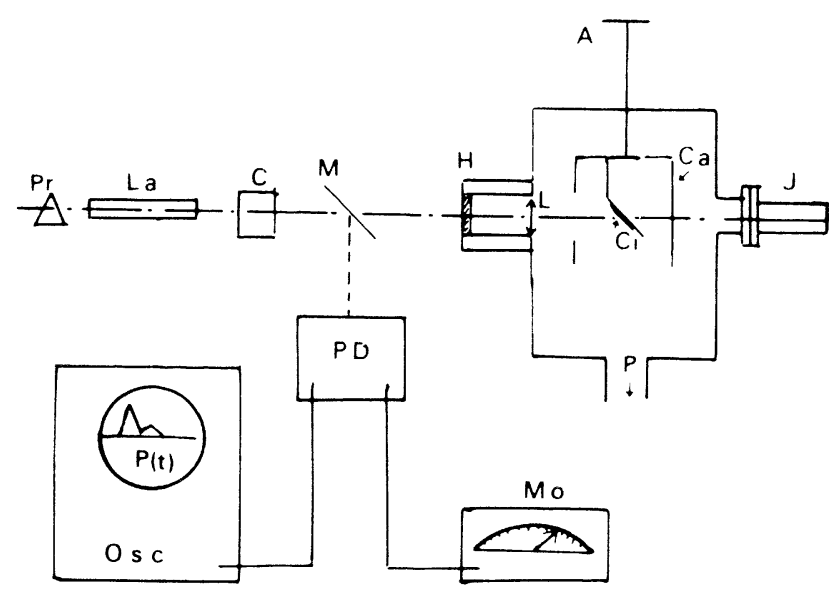

FIG. 1. - Dispositif expérimental.

$\mathrm{La}:$ le laser et son prisme tournant $\operatorname{Pr} ; \mathrm{C}$ : cuve à faces planes et parallèles optiquement travaillées, contenant les solutions aqueuses de sulfate de cuivre; $M$ : miroir semi-réfléchissant ; P. D. : photodiode ; Mo : enregistreur d'énergie ; Osc. : oscillographe Tecktronix 551 donnant $P(t) ; \mathrm{H}$ : hublot; $\mathrm{L}$ : lentille plan convexe $; \mathrm{Ci}$ : cible ; $\mathrm{A}$ : dispositif de commande manuelle de déplacement de la cible; $\mathrm{Ca}$ : cage métallique entourant la cible $; \mathrm{J}:$ jauge à ionisation de lecture du vide $; \mathrm{P}:$ orifice de pompage.

1. LE LASER. - Le laser utilisé est un laser à rubis, RDM 10, fourni par la Compagnie Générale d'Electricité. Le cristal a une longueur de $76 \mathrm{~mm}$ et un diamètre $\varnothing$ de $9,5 \mathrm{~mm}$. Ce laser déclenché au moyen d'un prisme tournant fournit, dans le rouge $(\lambda=6943 \AA)$ une impulsion lumineuse de $0,3 \mathrm{~J}$ dont la largeur à mi-hauteur peut atteindre $30 \mathrm{~ns}$. Cette durée d'impulsion correspond à une puissance maximale de $10 \mathrm{MW}$ et à une divergence angulaire

$$
\alpha=\frac{n^{2} \varnothing}{c \tau} \text { de } 5 \times 10^{-3} \mathrm{rd}
$$

$n=$ indice de réfraction du cristal de rubis,

$\tau=$ durée de l'impulsion lumineuse.

La vitesse de rotation du prisme est variable, elle permet d'allonger la durée de l'impulsion lumineuse jusqu'à $2 \mu \mathrm{s}$; l'énergie restant fixée à $0,3 \mathrm{~J}$ par une alimentation constante de la lampe à éclairs excitatrice.

Par ailleurs, pour une durée constante de l'émission lumineuse, on fait varier entre 0,3 et $0,003 \mathrm{~J}$ l'énergie transportée par le faisceau en l'atténuant au moyen d'une solution aqueuse de $\mathrm{CuSO}_{4}$ en concentration variable. Un miroir semi-réfléchissant envoie une partie du faisceau ( $2 \%$ ) sur une photodiode ; on obtient ainsi la structure temporelle de l'émission lumineuse $P(t)$ et, par intégration, l'énergie transportée en joule. La figure 2 représente les impulsions lumineuses obtenues pour différentes vitesses de rotation du prisme.
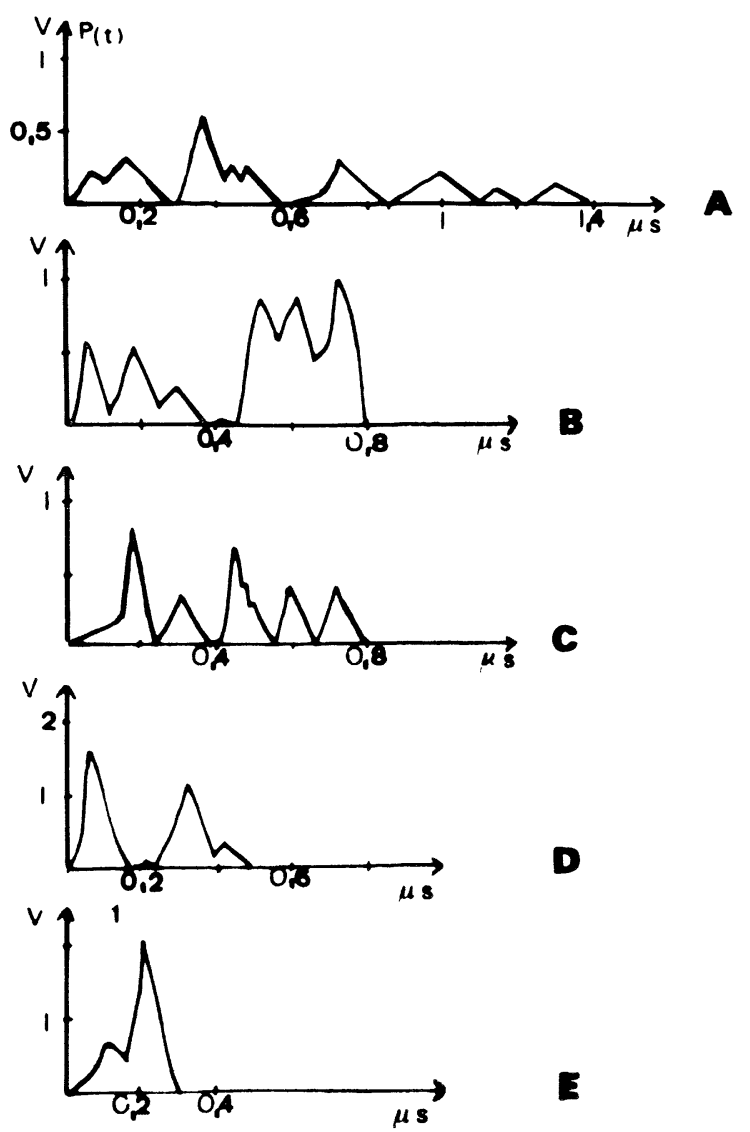

Fig. 2. - Répartition temporelle de l'énergie lumineuse.
Enregistrement

A
B
C
D
E

Vitesse de rotation du prisme (tours/s)

$\begin{array}{ll}100 & 250 \\ 200 & 280 \\ 250 & 290 \\ 300 & 300 \\ 400 & 290\end{array}$

2. Dispositif DE CONCENTRATION D'ÉNERgIE. - Le faisceau du laser pénètre par un hublot dans l'enceinte expérimentale. Il est focalisé sur la cible par une lentille convergente plan convexe, dont la distance focale est de $50 \mathrm{~mm}$. L'incidence moyenne du faisceau est de $45^{\circ}$. Le diamètre de la tache obtenue sur le plan focal de la lentille dépend essentiellement de la divergence angulaire du faisceau $\alpha, D=f \alpha \simeq 250 \mu$.

$$
D_{\max } \simeq 400 \mu \text { et } S \simeq 10^{-3} \mathrm{~cm}^{2} \text {. }
$$

L'éclairement de la tache n'est pas uniforme : il est très intense sur une zone centrale de diamètre $d_{1}=14 \mu$, limitée par le premier minimum de diffraction (spot focal) ; sa distribution dépend également de l'aberration de sphéricité latérale de la lentille $d_{2}$ voisine de 
$190 \mu$ (Fig. 3). De plus, l'émission lumineuse n'est pas constituée d'un train d'impulsions identiques, et l'on peut s'attendre aussi à un éclairement non uniforme dans le temps [7].

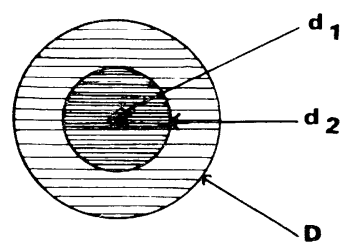

FIG. 3. - Répartition spatiale de l'énergie lumineuse.

Eclairement moyen. - En appelant $W$ l'énergie contenue dans le faisceau lumineux, nous définirons l'éclairement moyen $I_{\mathrm{m}}$ par le rapport $I_{\mathrm{m}}=W / S \tau$.

En première approximation, nous supposerons le flux lumineux uniformément réparti sur une surface $S$ de $10^{-3} \mathrm{~cm}^{2}$.

Dans nos conditions expérimentales :

$$
5 \times 10^{5}<I_{\mathrm{m}}<3 \times 10^{9} \mathrm{~W} / \mathrm{cm}^{2} .
$$

3. Méthode expérimentale. - Nous étudions différentes cibles métalliques polies : $\mathrm{Cu}, \mathrm{Ag}, \mathrm{Ti}, \mathrm{Au}$, $\mathrm{Ta}, \mathrm{Zr}, \mathrm{Pb}, \mathrm{Al}$, acier inox, placées dans une enceinte métallique, en acier inoxydable.

Un dispositif de pompage maintient une pression de $10^{-6} \mathrm{~mm}$ de $\mathrm{Hg}$. La cible est placée à l'intérieur d'une cage métallique. La mesure du vide est effectuée au moyen d'une jauge à ionisation, située hors d'atteinte des particules neutres ou chargées produites au cours de l'interaction.

Nous effectuons des tirs laser sur la cible en présentant chaque fois une surface vierge au faisceau lumineux, à l'aide d'un dispositif à crémaillère permettant une translation de la cible dans le plan focal de la lentille. Après irradiation de la cible, les dégâts produits par l'impact sont examinés au microscope et au microcomparateur. Ce dernier appareil permet un enregistrement du profil géométrique de la sur̃face soumise à l'irradiation avec une sensibilité meilleure que le micron.

III. Résultats. - Les dégâts observés dépendent des propriétés de l'impulsion lumineuse et des propriétés physiques de la cible.

1. Influence de L'ÉClairement moyen $I_{\mathrm{m}}$. - Dans ces expériences, nous fixons les paramètres du laser et nous faisons varier l'éclairement $I$ au moyen des solutions de $\mathrm{CuSO}_{4}$. La répartition de l'éclairement, représentée sur la figure 3 , reste donc constante.

1. a Quelle que soit la nature de la cible, pour un éclairement moyen $I_{\mathrm{m}}<10^{6} \mathrm{~W} / \mathrm{cm}^{2}$, on n'observe aucun dégât, mais il se produit une élévation de température. Les variations de pression observées dans l'enceinte ainsi que l'étude des particules émises, prouvent qu'il y a désorption et ionisation des espèces neutres fixées superficiellement [8]. Dans certains cas. il apparaît sur la surface des teintes sombres, caractéristiques d'une oxydation due à un échauffement local en présence de l'oxygène résiduel de l'enceinte.

1. $b \mathrm{Si}$ on augmente progressivement l'éclairement, au voisinage d'un certain seuil, $I_{0} \simeq 10^{6} \mathrm{~W} / \mathrm{cm}^{2}$, légèrement inférieur dans le cas du plomb, on commence à apercevoir des dégâts localisés au centre de la tache, sur une zone de diamètre égal à $100 \mu$. Cette zone n'est pas altérée d'une façon homogène comme le montrent la micrographie et l'enregistrement du profil de la surface (Fig. 4).

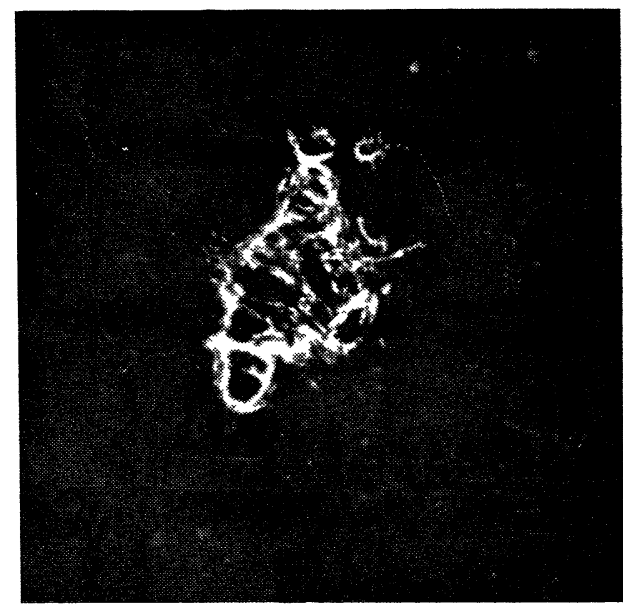

FIG. 4. - Allure des dégâts au voisinage du seuil.

Cible de cuivre : $I_{m}=4 \times 10^{6} \mathrm{~W} / \mathrm{cm}^{2}$

Echelles : micrographie :

$18 \mathrm{~mm}=100 \mu$

L'éclairement non uniforme dans le temps et dans l'espace doit conduire à un échauffement non uniforme ; d'autre part, des défauts superficiels de la cible peuvent entraîner une absorption plus grande du faisceau lumineux.

1. $c$ Pour des éclairements $I$ tels que

$$
10^{6}<I_{\mathrm{m}}<10^{7} \mathrm{~W} / \mathrm{cm}^{2},
$$

on observe une zone fondue superficielle couvrant toute l'étendue de la tache focale. Après examen au microcomparateur, elle se présente sous la forme d'une succession de hauteurs et de dépressions (Fig. 5).

Cette structure chaotique peut s'expliquer par la grande rapidité du chauffage et du refroidissement de la cible. La surface, brusquement solidifiée, présente l'aspecî d'une succession de vagues figées.

1. $d$ Pour des éclairements $I>10^{7} \mathrm{~W} / \mathrm{cm}^{2}$, les dégâts sont encore plus marqués et présentent alors l'allure de véritables cratères. La zone endommagée occupe une surface qui croît avec l'éclairement. On observe au centre une dépression entourée de parois abruptes qui dépassent le niveau initial de la cible, et 


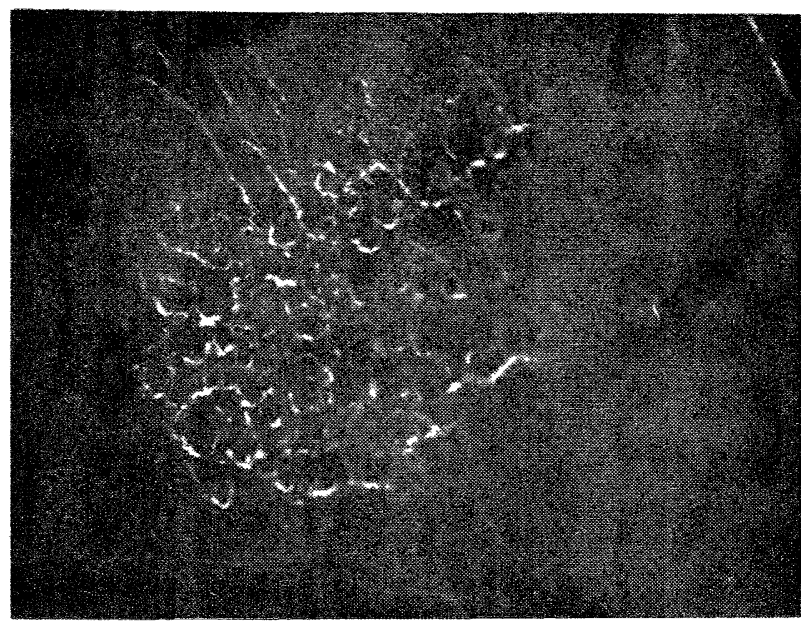

Fir, § - tllure des dégâts pour un éclaırement légeıement supérieur au seuil.

Cible tion $L_{-\mathrm{n}}=10^{\circ} \mathrm{W} \mathrm{cm}^{-}$

mucrographie

$18 \mathrm{~mm}-100 \mu$

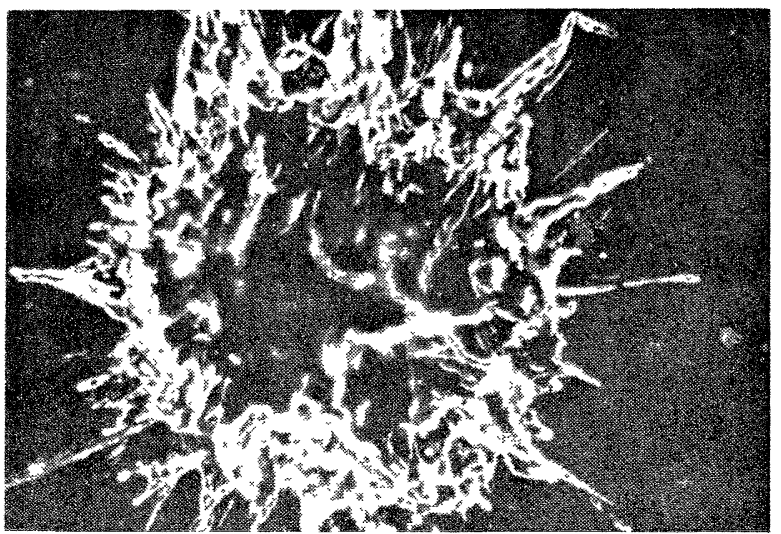

Fu 6 - Allure dew crateres

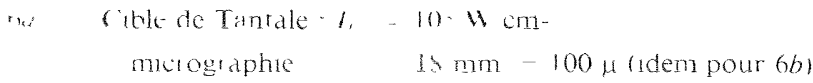

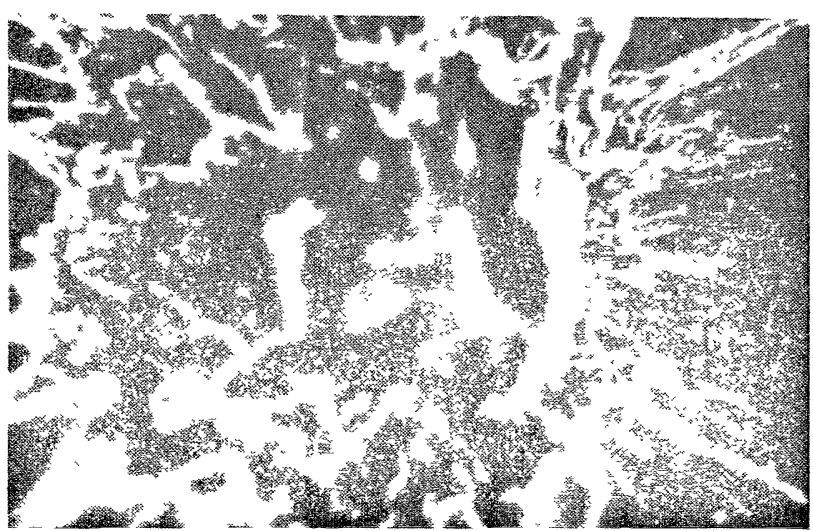

ht. - Cible de Zroonium I $=10 \cdot 11 \mathrm{~m}$ au-delà on aperçoit des particules solides condensées en aigrettes (Fig. 6).

Au moment de linteraction. il doit $y$ aroir une fusion superficielle accompagnée d'une éruption du liquide courrant la surface centrale. L’écoulement du metal fondu sur les bords de la zone centrale s ient former les parois surélesées du cratère et les aigrettes proviennent de la projection de gouttelettes hors du cratère

1. e Une augmentation ultérieure d'éclarement $I>10^{9} \mathrm{~W} \cdot \mathrm{cm}^{2}$ ne modifie pas l'allure générale des dégâts obserrés, mais les aigrettes s'étendent sur une plus grande surface.

2. Influence des paramėtres du faisceal LUUiNELX. - Nous étudions l'évolution des cratères en fonction de l'éclairement en opérant, solt à énergie lumineuse constante, en faisant varier la durée de l'impulsion lumineuse $\tau$, soit à $\tau$ constant en faisant rarıer l'énergie $W$ contenue dans le faisceau au moyen des solutions aqueuses de $\mathrm{CuSO}_{4}$. Nous caractérisons par $\varnothing_{\mathrm{c}}$ et $\approx_{\mathrm{c}}$ le diamètre et la profondeur du cratère

a) Influence de la durée $\tau$ de l'impulsion lumineuse. Nous avons rassemblé dans la figure $7 a$ les résultats relatifs à cette étude On remarque que $\varnothing$ augmente quand $t$ diminue. La diminution de la durée d'impulsion $\tau$ a pour conséquence l'augmentation de la dirergence $x$ du faisceau, donc du diamètre $D=f y$ de la tache lumineuse et par suite du diamètre du cratère

Quant à la profondeur $z_{\mathrm{c}}$, d'une manière générale. elle diminue avec $\tau$. Deux cas font exception $\left(T_{i}(70 \mathrm{~mJ})\right.$ et $\mathrm{Al}(100 \mathrm{~mJ})), z_{\mathrm{c}}$ cioît. passe par un maximum et décroît : nous remarquons que ce comportement correspond à des énergies lumineuses relativement faibles $(70$ et $100 \mathrm{~mJ}$ ), et peut sexpliquer par les pertes d'énergie par conduction dans la cible qui augmentent arec la durée t. comme nous le verrons plus loin.

b) Influence de l'energie contenue dans le tansceun. Les résultats relatifs à cette étude sont rassemblés dans la figure $7 b$ t restant constant. on peut admettre que la surface éclairée reste constante.

On remarque que $\varnothing$, et $Z$, augmentent rapidement avec lénergie à partir d'une valeur de l'éclaırement comprise entre $10^{\circ}$ et $10^{*} \mathrm{~W} \mathrm{~cm}^{2}$. La figure $7 b$ montre la variation de $\varnothing$, et $Z$, pour différents matériaux la durée d’impulsion étant la même $(\tau=0.4 \mu$ s). alle met en éridence l"influence des proprićtés physiques de la cible.

La figure $7 c^{\circ}$ montre l'influence de la durée de lirrddiation pour une cible donnée. La croissance de $\varnothing$. et de $Z_{\mathrm{c}}$ avec leénergie est constante pour des durées dimpulsion $\tau \geqslant 0.8 \mu \mathrm{s}$; par contre s1 $\tau<0,8 \mu \mathrm{s}$ cette croissance de $\varnothing_{\text {c }}$ et $Z$, avec l'énergle est beaucoup plus faible aux fortes énergies.

Pour une cible donnée on constate que les dimensions des cratères augmentent avec la durée de l'irradiatıon pour des valeurs de léclarement $I<10^{9} \mathrm{~W} \mathrm{~cm}^{2}$. ensulte 1 apparait un phénomène de saturation, sen- 


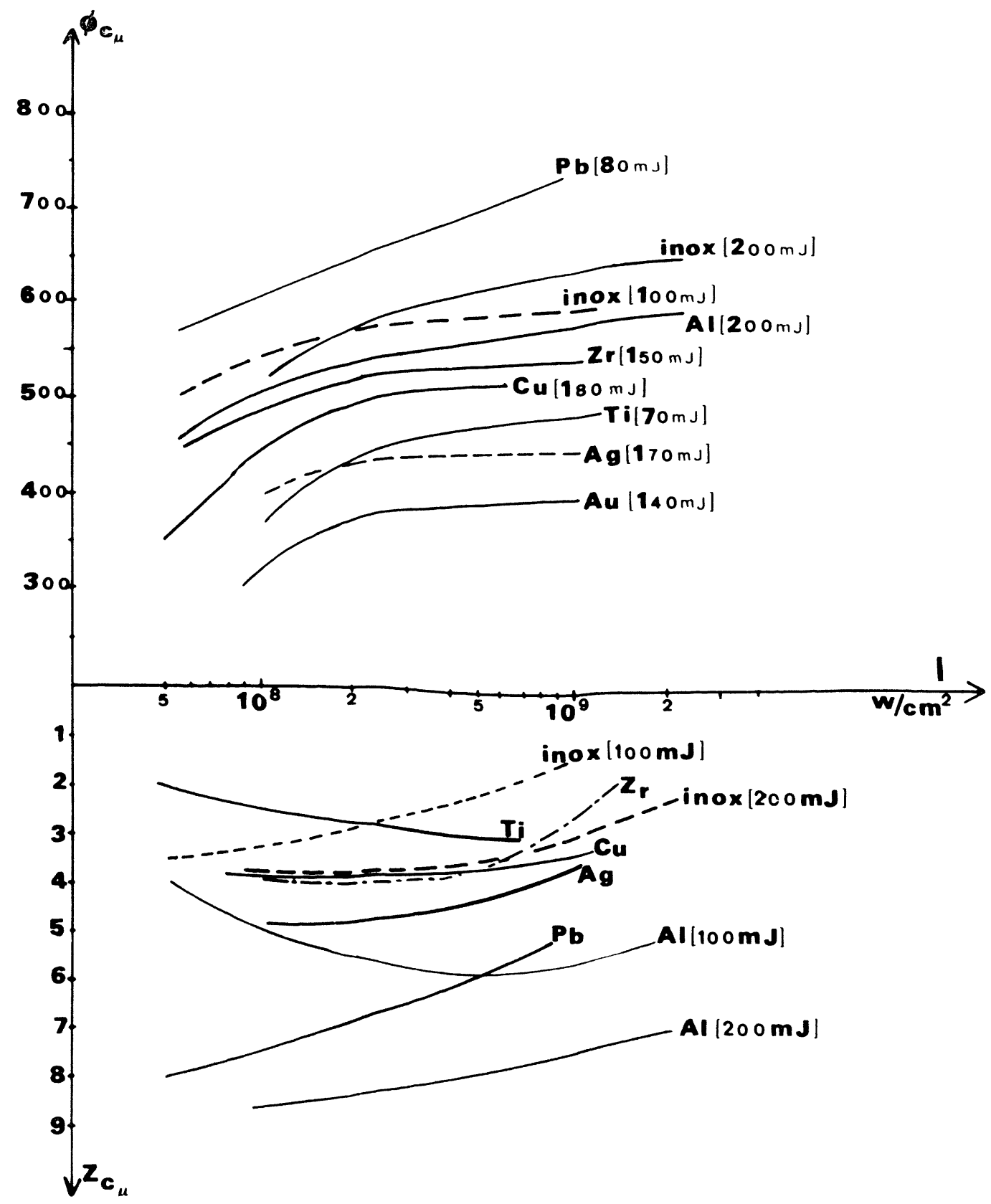

FIG. 7. - Evolution des cratères en fonction de l'éclairement moyen $I_{m}$.

7a. - Influence de la durée de l'impulsion lumineuse à énergie constante (valeur de cette énergie).

sible pour les courtes durées d'impulsion, comme si l'énergie présente dans le faisceau lumineux pénétrait de moins en moins dans la cible.

3. INFLUENCE DES PROPRIÉTÉS PHYSIQUES DE LA SURFACE. - 3.a Influence du pouvoir réflecteur de la surface. Nous avons opéré avec des cibles de cuivre présentant différents états de surface : brut de laminage, poli et corrodé à l'acide chlorhydrique. Pour toutes ces expériences nous avons conservé une durée d'impulsion constante, en faisant varier l'éclairement à l'aide de solutions de $\mathrm{CuSO}_{4}$.

- Quand on altère le pouvoir réflecteur de la surface par destruction mécanique ou chimique du poli, on constate que le seuil d'apparition des dégâts est beaucoup plus faible $\left(<\right.$ à $10^{6} \mathrm{~W} / \mathrm{cm}^{2}$ ) pour le $\mathrm{Cu}$.

- Au-dessus du seuil : l'intensité des dégâts, pour un même éclairement, est plus importante pour la surface non polie que pour la surface polie (tableau I).

\section{TABleau I}

\begin{tabular}{|c|c|c|c|}
\hline$\underset{\mu \mathrm{s}}{\tau=0,4}$ & $\underset{\mathrm{mJ}}{\mathrm{WmJ}}$ & $\begin{array}{c}I \\
\text { Watt } / \mathrm{cm}^{2}\end{array}$ & $\varnothing_{c} \mu$ \\
\hline brut & 170 & $4,2 \times 10^{8}$ & 450 \\
\hline polie & 180 & $4,5 \times 10^{8}$ & 450 \\
\hline
\end{tabular}

- On a comparé les dégâts provoqués sur une cible polie par un seul tir laser à ceux que provoquent deux 


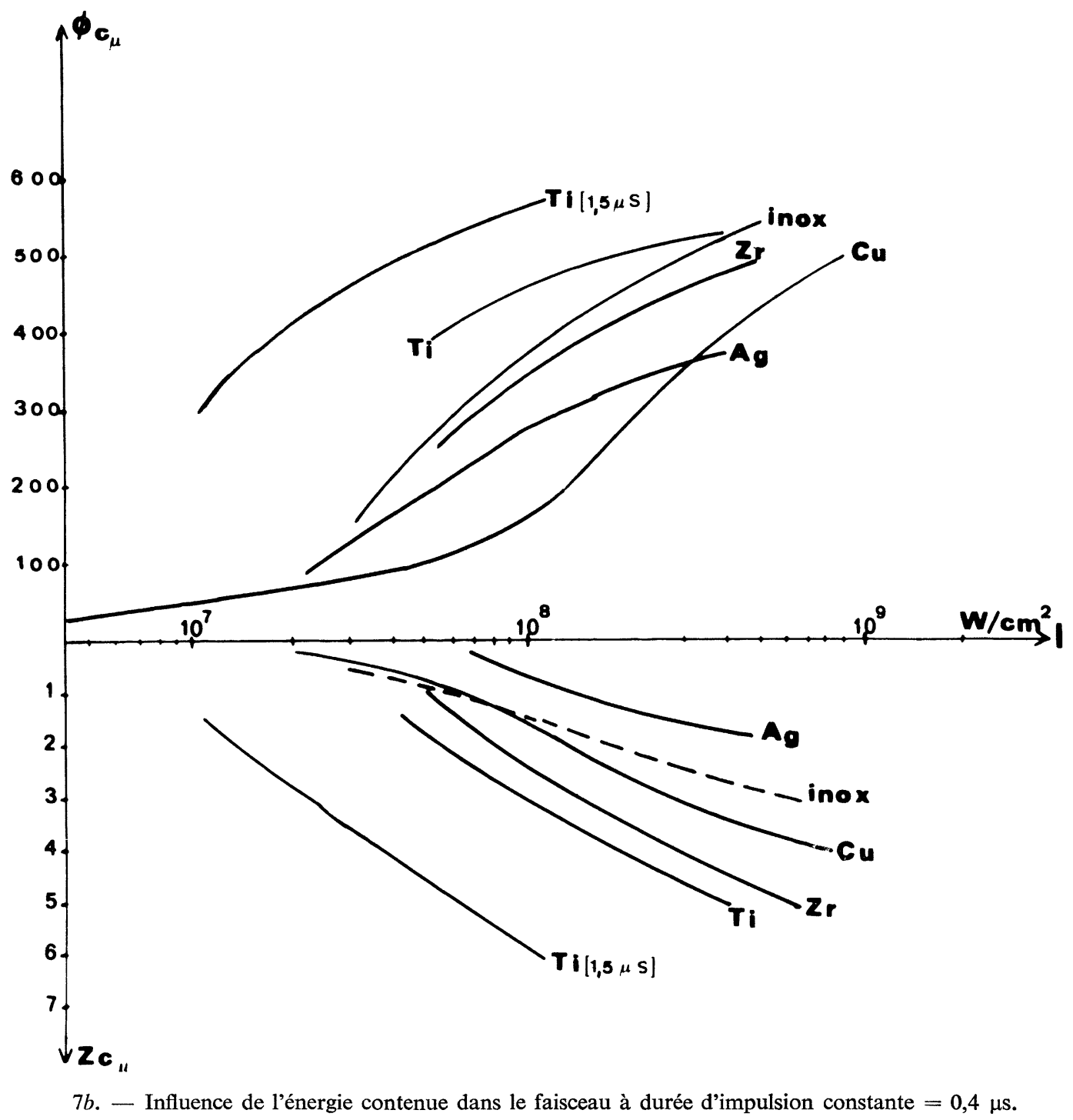

tirs de plus faibles éclairements. On observe que l'étendue et la profondeur des dégâts sont toujours plus importantes dans le second cas, alors que l'énergie totale reçue par la cible est au plus égale à l'énergie contenue dans le tir unique.

Même si le premier des deux tirs faibles crée peu de dégâts, il suffit pour altérer le pouvoir réflecteur et exalter l'effet du second tir.

- Le tableau II donne la profondeur du cratère obtenu par des impacts multiples sur la même surface de cuivre, dans différentes conditions d'éclairements mais pour des énergies lumineuses voisines.
Nous avons représenté sur la figure 8 la profondeur relative, $z_{\mathrm{r}}$, du cratère en fonction du nombre $N$, de tirs consécutifs sur la même surface. L'unité de longueur est la profondeur de cratère atteinte par un seul impact $(3$ à $4 \mu)$.

La profondeur mesurée en unités relatives n'est pas proportionnelle au nombre d'impacts.

On observe un effet d'exaltation pour des valeurs de $\tau \geqslant 0,8 \mu \mathrm{s}$ (A et B) et un effet inverse si $\tau<0,8 \mu \mathrm{s}$ (C et D). Dans ce dernier cas, correspondant à de faibles valeurs de $\tau$, nous nous trouvons dans un domaine d'éclairement très élevé où les processus de

\section{TABLEAU II}

\begin{tabular}{|c|c|c|c|c|c|c|c|}
\hline Cratère & $\begin{array}{c}\tau \\
\mu \mathrm{s}\end{array}$ & $\begin{array}{l}\text { Nombre } \\
\text { d'impacts }\end{array}$ & $\begin{array}{c}\text { Energie } \\
\text { moyenne par } \\
\text { impact en } \mathrm{mJ}\end{array}$ & $\begin{array}{l}\text { Energie } \\
\text { totale } \\
\text { en } \mathrm{mJ}\end{array}$ & $\begin{array}{l}I_{\mathrm{m}} \text { en } \\
\mathrm{W} / \mathrm{cm}^{2}\end{array}$ & $\begin{array}{c}z_{\mathrm{c}} \\
\mathrm{en} \mu\end{array}$ & $\begin{array}{l}\varnothing_{\mathrm{c}} \\
\text { en } \mu\end{array}$ \\
\hline A & 1,5 & 5 & $150 \mathrm{~mJ}$ & $\overline{780}$ & $10^{8}$ & $\overline{30}$ & 400 \\
\hline B & 0,8 & 4 & 160 & 650 & $2 \times 10^{8}$ & 18 & 450 \\
\hline $\mathrm{C}$ & 0,4 & 8 & 150 & 1200 & $4 \times 10^{8}$ & 20 & 500 \\
\hline $\mathrm{D}$ & 0,2 & 9 & 140 & 1200 & $7 \times 10^{8}$ & 12 & 500 \\
\hline
\end{tabular}




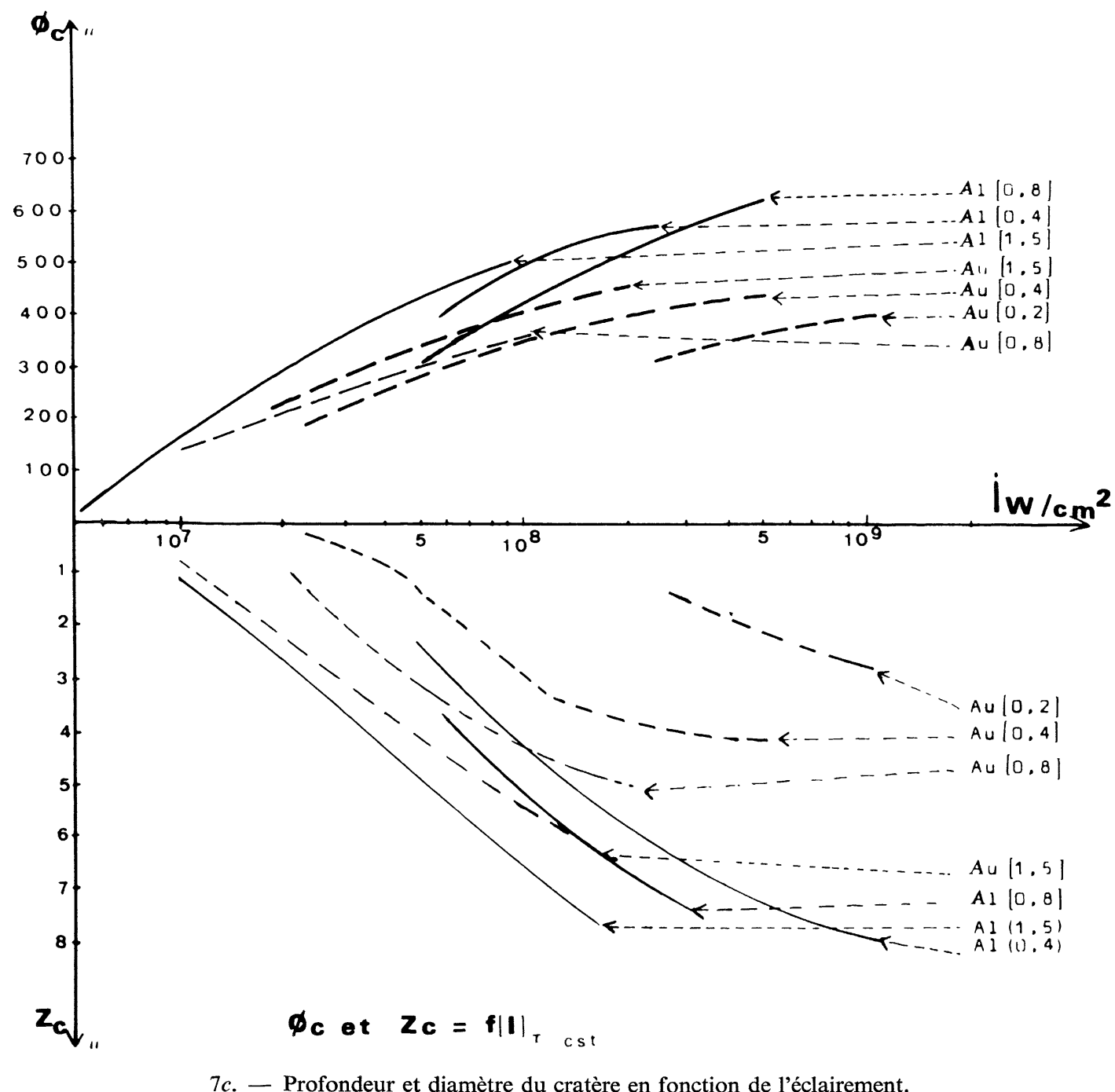

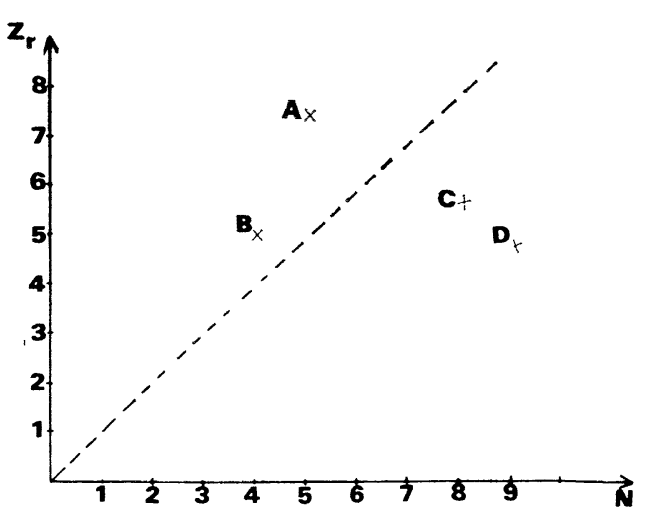

Fig. 8. - Profondeur relative du cratère, $Z_{r}$, en fonction du nombre de tirs consécutifs, $N$, sur la même surface.

vaporisation sont moins efficaces que dans les cas $\mathrm{A}$ et $\mathbf{B}$ comme nous le verrons plus loin. L'interaction laser conduit à une pression très élevée au sein de la vapeur formée ; une partie de la phase liquide formée reste $\mathrm{au}$ fond du cratère où elle se solidifie.

3.b Influence des propriétés thermiques. - Nous avons opéré avec des matériaux très différents et nous avons étudié l'influence de la température de fusion $T^{\circ} f$, de la tension de vapeur au point de fusion $P_{f}$, de la diffusivité thermique $k=K / C, K$ et $C$ étant respectivement la conductivité thermique et la capacité calorifique, et de l'admittivité $\sqrt{K C}$ du matériau pour des conditions d'éclairement identiques. Les résultats sont rassemblés dans le tableau III.

La dépendance des dégâts occasionnés avec les propriétés thermiques est peu apparente. Toutefois, c'est avec le plomb que l'on observe les plus gros dégâts.

Malheureusement, les paramètres diffusivité thermique et température de fusion ont tous deux des valeurs faibles et l'on ne peut discerner leur influence respective.

4. INFLUENCE DE LA PRESSION DANS L'ENCEINTE. Nous notons que les cratères sont moins marqués, pour des conditions d'éclairement identiques et pour un même matériau, si l'on opère à la pression atmosphérique (Fig. 9). Ceci peut être dû aux pertes thermiques plus importantes ou bien à un effet d'écran 


\begin{tabular}{|c|c|c|c|c|c|c|c|c|c|c|}
\hline \multicolumn{11}{|c|}{ TABLEAU III } \\
\hline Cible & & $\mathrm{Pb}$ & $\mathrm{Al}$ & $\mathrm{Ag}$ & $\mathrm{Au}$ & $\mathrm{Cu}$ & Inox & $\mathrm{Ti}$ & $\mathrm{Zr}$ & $\mathrm{Ta}$ \\
\hline$T^{0} f$ & & $\overline{327^{\circ}}$ & $\overline{660}$ & $\overline{961^{\circ}}$ & $1 \overline{063^{\circ}}$ & $1 \overline{083^{\circ}}$ & $\begin{array}{c}\overline{-} \\
\text { env. } \\
1550^{\circ}\end{array}$ & $1 \overline{690^{\circ}}$ & $1 \overline{750^{\circ}}$ & $2 \overline{99}^{\circ}$ \\
\hline$K$ en $\mathrm{W} \cdot \mathrm{cm}^{-1}$ & $\mathrm{~d}^{\circ} \mathrm{K}^{-1}$ & 0.34 & 1,7 & 4,18 & 2,96 & 3,88 & 0,75 & 0,15 & 0,5 & 0,54 \\
\hline$C$ en $\mathrm{J} / \mathrm{cm}^{-3}$ & $\mathrm{~d}^{\circ} \mathrm{K}^{-1}$ & 1.42 & 2,43 & 2.47 & 2,51 & 3,37 & 3,5 & 2,5 & 5,6 & 2,46 \\
\hline$k$ en $\mathrm{cm}^{2}$ & & 0.24 & 0.91 & 1.71 & 1.18 & 1,14 & 0.21 & 0,06 & 0,09 & 0,23 \\
\hline $\begin{array}{l}\sqrt{K C} \text { en } \\
\mathrm{W}: \mathrm{cm}^{-2} \times \mathrm{s}^{1}=\end{array}$ & $\cdot \mathrm{d}^{\circ} \mathrm{K}^{-1}$ & 0,7 & 1,56 & 3,2 & 2.73 & 3,62 & 1,04 & 0,6 & 1,67 & 1,15 \\
\hline$P \mathrm{~mm} \mathrm{Hg}$ & & $4 \div 10^{-9}$ & $3 、 10^{-9}$ & $3<10^{-3}$ & $2 \times 10^{-5}$ & $4 \times 10^{-4}$ & $2 \times 10^{-2}$ & $4 \times 10^{-3}$ & $2 \times 10^{-5}$ & $6 \times 10^{-3}$ \\
\hline$\tau=0.8 \mu \mathrm{s}$ & $z_{\mathrm{e}}$ & 10 & 5 & 3 & 3 & 2,5 & 4,5 & 2 & 5 & 3 \\
\hline$W=100 \mathrm{~mJ}$ & $\begin{array}{l}\mu \\
\mu \\
\mu\end{array}$ & 700 & 500 & 400 & 400 & 400 & 500 & 450 & 450 & 400 \\
\hline
\end{tabular}

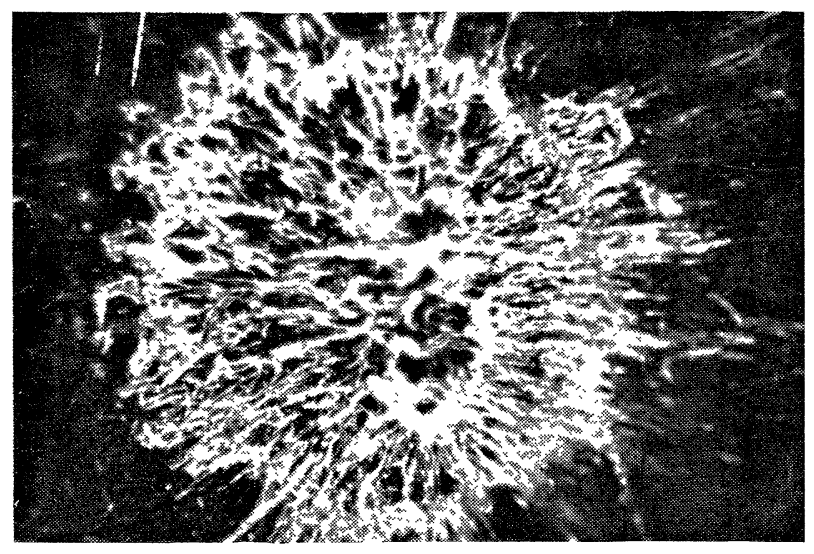

FIG. 9. - Influence de la pression dans l'enceinte. Cible de cuivre; $I_{m}=10^{8} \mathrm{~W} / \mathrm{cm}^{2}$. $9 a .-P=1$ Atm.

micrographie $\quad 18 \mathrm{~mm}=100 \mu$ (idem pour $9 b)$

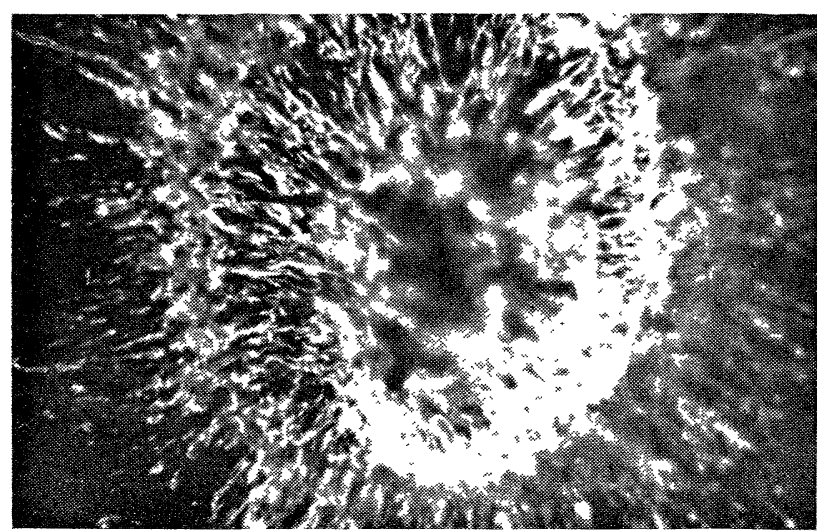

9b. $-\mathrm{P}=10^{-5} \mathrm{~mm} \mathrm{Hg}$.

du plasma d'air provoqué par ionisation par les particules énergétiques en provenance de la cible.

D'autre part, la perte de poids de la cible est plus faible à l'air libre quà la pression de $10^{-6} \mathrm{~mm} \mathrm{Hg}$; cette perte, à l'air libre, est de l'ordre de $80 \mu \mathrm{g}$ pour 50 impulsions de $1,5 \mu$ s chacune, correspondant au total à $6,5 \mathrm{~J}$ et dans le cas d'une cible de Tantale.

De plus, la présence d'air favorise les attaques chimiques à haute température. C'est ainsi que l'on observe des auréoles noires autour des cratères d'une cible en acier inoxydable. Pour le cuivre, les auréoles sont rouges ou noires, suivant la pression de l'enceinte.

5. QUANTITÉS DE SUbSTANCE ÉJECTÉE. - Nous avons essayé de déterminer par deux méthodes les quantités de substance perdue par la cible :

a) Par comparaison à partir des enregistrements effectués au microcomparateur entre le volume $V_{1}$ du cratère correspondant à une disparition de matière, et le volume $V_{2}$ des bords situés au-dessus du niveau initial (apparition de matière) (Fig. 10). La différence $V_{1}-V_{2}$ représente la quantité de substance vaporisée, compte non tenu des aigrettes. Dans tous les cas, on trouve une masse $\mathrm{m}$ de l'ordre de $10^{-6} \mathrm{~g}$ pour un impact.

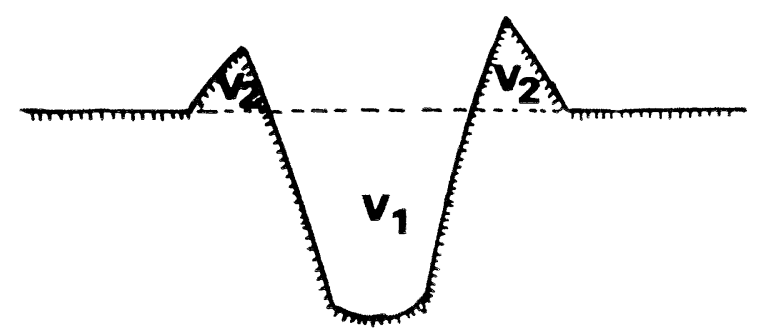

FIG. 10. - Volume de substance vaporisée. $V=V_{1}-V_{2}$.

b) Par pesée en déterminant la masse perdue par la cible, après l'avoir soumise à un grand nombre de tirs :

- Le tableau IV montre, dans le cas d'une cible de Tantale, que la quantité de substance vaporisée diminue avec la durée $\tau$ de l'impulsion lumineuse, ce qui est en accord avec les résultats obtenus dans l'étude des dimensions des cratères.

III. Interprétation - modèle théorique. - 1. INTRODUCTION. - Si $I_{0}$ désigne le flux lumineux incident sur la surface métallique dont le pouvoir réflecteur est $R$ pour la longueur d'onde considérée, le flux lumineux absorbé est

$$
I=I_{0}(1-R)=I_{0} A .
$$

Le pouvoir absorbant $A$, lié aux propriétés élec- 
TABLEAU IV

\begin{tabular}{|c|c|c|c|c|c|c|c|c|}
\hline Cible & $\begin{array}{c}\text { Nombre } \\
\text { d'impacts }\end{array}$ & $\begin{array}{c}\text { Energie } \\
\text { totale } \\
\mathrm{J}\end{array}$ & $\begin{array}{c}\text { Masse } \\
\text { totale } \\
\text { vaporisée }\end{array}$ & $\begin{array}{c}\tau \\
\mu \mathrm{s}\end{array}$ & $\underset{\mathrm{W} / \mathrm{cm}^{2}}{I}$ & $\begin{array}{c}\text { Energie } \\
\text { moyenne } \\
\text { par } \\
\text { impact } \\
\text { mJ }\end{array}$ & $\begin{array}{c}\text { Masse } \\
\text { vaporisée } \\
\text { par } \\
\text { impact } \\
\mu \mathrm{g}\end{array}$ & $\begin{array}{c}\text { Masse } \\
\text { vaporisée } \\
\text { par } \\
\text { Joule } \\
\mu \mathrm{g}\end{array}$ \\
\hline \multirow[t]{2}{*}{ Ta } & 100 & 10 & 200 & 1,5 & $7 \times 10^{7}$ & 100 & 2 & 20 \\
\hline & 224 & 13,3 & 155 & 0,8 & $8 \times 10^{7}$ & 60 & 0,6 & 12 \\
\hline$P=10^{-6}$ & 560 & 48 & 436 & 0,4 & $2 \times 10^{8}$ & 85 & 0,78 & 9,1 \\
\hline $\mathrm{mm} \mathrm{Hg}$ & 358 & 22 & 142 & 0,2 & $3 \times 10^{8}$ & 62 & 0,4 & 6,4 \\
\hline
\end{tabular}

troniques du matériau, est en relation inverse avec la mobilité des électrons de conduction; mais pour une même substance ce n'est pas une donnée bien définie : elle varie en particulier avec l'état physicochimique de la surface et sa température. L'altération de la surface ainsi qu'une augmentation de la température entraînent toujours une augmentation du pouvoir absorbant.

- L'étude de la propagation d'une onde électromagnétique dans un conducteur montre que l'atténuation de cette onde est importante et que la pénétration au-delà de la surface est très faible, en pratique dans une peau d'épaisseur trois à quatre fois $z_{0}$.

$$
z_{0}=\frac{\lambda}{2 \pi n_{\mathrm{e}}}
$$

$\mathrm{n}_{\mathrm{e}}$ caractéristique du conducteur considéré est appelée l'indice d'extinction, $z_{0}$ représente l'épaisseur pour laquelle l'intensité est divisée par $e^{2}, z_{0} \simeq 150 \AA$ pour le cuivre.

2. TRANSFORMATION DE L'ÉNERGIE ÉLECTROMAGNÉTIQUe EN CHALEUR DANS LE MATÉRIAU. - Quand l'onde pénètre dans le conducteur, les électrons de conduction oscillent sous l'effet du champ électrique $E$ incident; si l'électron était isolé, son énergie d'oscillation demeurerait en moyenne nulle, mais étant donné la densité très importante des particules au sein de la cible, il se produit de nombreuses collisions pendant lesquelles les électrons acquièrent une partie de l'énergie du rayonnement et en cèdent aux atomes constituant le réseau : il en résulte un échauffement du milieu.

Cet échauffement s'effectue en un temps très bref, de l'ordre de $10^{-12} \mathrm{~s}$ d'après F. Seitz [10]; puis la chaleur produite dans la zone d'interaction diffuse par conduction d'une manière isotrope et d'autant plus facilement que le milieu est meilleur conducteur. Si $k$ est la diffusivité thermique du matériau, on caractérise la pénétration de la chaleur par l'ordonnée $z_{\mathrm{e}}$ du front thermique en expansion :

$$
z_{\mathrm{e}}=(4 k t)^{1 / 2}
$$

qui représente en même temps l'épaisseur chauffée pendant l'intervalle de temps $t$.
En particulier, pour une durée de temps $t$ égale à la durée de l'impulsion lumineuse, $t=\tau$.

$$
2 \times 10^{-7} \mathrm{~s} \leqslant t=\tau \leqslant 1,5 \times 10^{-6} \mathrm{~s}
$$

pour la plupart des métaux $k$ est tel que

$$
\begin{gathered}
10^{-1}<k<1 \mathrm{~cm}^{2} \mathrm{~s}^{-1} \\
\text { et d'après (1) } 6 \times 10^{-4} \mathrm{~cm} \leqslant z_{\mathrm{e}} \leqslant 2 \times 10^{-3} \mathrm{~cm} \text { d'où } \\
z_{\mathrm{e}} \gg z_{0} .
\end{gathered}
$$

On voit que le front thermique pénètre la cible sur une profondeur de quelques microns, tandis que l'onde lumineuse ne pénètre que de quelques centaines d'angstrœms.

La pénétration du front thermique s'accompagne évidemment d'une modification des propriétés physiques de la cible, mais on est conduit à les négliger en première approximation.

Ainsi, d'après Carslaw et Jaeger [11], sous l'effet du choc thermique $I_{\mathrm{a}} t^{1 / 2}, I_{\mathrm{a}}$ étant le flux énergétique absorbé, l'évolution de la température d'un solide à la profondeur $z$ et au temps $t$ est donnée par :

$\Delta \theta=\frac{2 I_{\mathrm{a}}}{K}\left[\left(\frac{k t}{\pi}\right)^{1 / 2} \cdot \exp \left[-\frac{z^{2}}{4 k t}\right]-\frac{z}{2} \operatorname{erf} c \frac{z}{2 \sqrt{k t}}\right]$.

Au temps $t=\tau$, la température superficielle $(z=0)$ sera maximale et donnée par :

$$
\theta_{c}^{0}=\frac{2 I_{\mathrm{a}}}{k}\left(\frac{k \tau}{\pi}\right)^{1 / 2}=\frac{2}{\sqrt{\pi}} \frac{W_{\mathrm{a}}}{S \sqrt{\tau}} \times \frac{1}{\sqrt{K C}}
$$

en supposant une température initiale voisine de $0^{\circ} \mathrm{C}$, $W_{\mathbf{a}}$ étant l'énergie lumineuse absorbée par la surface $S$ pendant la durée de temps $\tau$; $K$ et $k$ étant respectivement la conductibilité et la diffusivité thermique à la température de la cible avant l'impact photonique.

D'après cette formule, on doit s'attendre à une variation considérable de l'échauffement en fonction de la répartition spatiale $(S)$ et temporelle $(\tau)$ de l'énergie lumineuse incidente.

Remarque. - Nous avons négligé dans ce calcul, la présence des phases liquide et gazeuse, ainsi que la puissance rayonnée par la surface qui n'est que de quelques centaines de $\mathrm{W} / \mathrm{cm}^{2}$ aux températures supposées atteintes (quelque $10^{3} \mathrm{~K}$ ). 
3. INFLUENCE DES PARAMÈTRES LUMINEUX SUR L'ÉCHAUFFEMENT. - a) Influence de la durée de l'impulsion lumineuse $\tau$. - Pour une même énergie incidente $W$, plus $\tau$ sera grand, moins la température atteinte sera importante mais plus la profondeur chauffée $z_{\mathrm{e}}=(4 k \tau)^{1 / 2}$ sera grande. Si l'énergie contenue dans le faisceau est suffisante pour entraîner la vaporisation, l'épaisseur vaporisée augmentera avec $\tau$ (Fig. 7a). Par contre si l'énergie est insuffisante pour entretenir la vaporisation, elle sera perdue par conduction au sein de la cible (Fig. $7 a$ cas de $\mathrm{Al}(100 \mathrm{~mJ})$ et de $\mathrm{Ti}$ $(70 \mathrm{~mJ}))$; les pertes par conduction sont d'autant plus importantes que le milieu est meilleur conducteur.

Inversement, si $\tau$ est faible, la température atteinte par la surface est importante, la profondeur chauffée diminue et les pertes par conduction sont d'autant moins importantes que le milieu est moins bon conducteur.

b) Influence de l'énergie contenue dans le faisceau. Pour une durée d'impulsion $\tau$ donnée, la température superficielle croît avec l'énergie $W_{\mathrm{a}}$, tant que l'énergie est insuffisante pour produire un échauffement susceptible d'entraîner la vaporisation $\left(I \leqslant 3 \times 10^{7} \mathrm{~W} / \mathrm{cm}^{2}\right)$, les dégâts sont faibles (Fig. $7 b$ ) et on n'observe pas de pertes de poids caractéristiques.

Pour les valeurs supérieures de l'énergie, la vaporisation devient importante, $\varnothing_{\mathrm{c}}$ et $Z_{\mathrm{c}}$ augmentent rapidement avec l'énergie suivant la loi $Z_{\mathrm{c}}=f(W)^{\alpha}$ avec $\alpha=0,6$.

Puis pour des valeurs de l'énergie telles que $I \geqslant 4 \times 10^{8} \mathrm{~W} / \mathrm{cm}^{2}, \varnothing_{\mathrm{c}}$ et $Z_{\mathrm{c}}$ croissent moins vite avec l'énergie $W, \alpha<0,6$; et ceci est d'autant plus sensible que la durée de l'impulsion lumineuse est faible (Fig. 7c). L'énergie lumineuse contenue dans le faisceau est supérieure à celle nécessaire pour chauffer et vaporiser la substance; la surface de la cible est chauffée à une température bien supérieure à sa température de vaporisation, les particules vaporisées sont plus énergétiques et le rendement de vaporisation $\rho_{\mathrm{v}}$ décroît :

$$
\left(\rho_{\mathrm{v}}=\frac{\text { énergie utilisée pour la vaporisation }}{\text { énergie contenue dans le faisceau }}\right) .
$$

4. INFLUENCE DES PROPRIÉTÉS PHYSIQUES DU MATÉRIAU. - a) Influence des propriétés thermiques. $W, S, \tau$, restant constants, nous voyons sur la formule (3) que la température superficielle atteinte est une fonction décroissante de $\sqrt{K C}$. Ce produit appelé "admittivité thermique» du matériau, caractérise le flux de chaleur qui pénètre dans la cible à partir de la surface. Plus ce produit est élevé, moins l'échauffement superficiel est important; mais par contre, il se fait ressentir dans les couches plus profondes de la cible. D'autre part, comme l'onde électromagnétique pénètre plus facilement dans un milieu lorsque celui-ci est moins conducteur, le même faisceau lumineux induira de plus gros dégâts sur une cible peu conduc- trice que sur une cible bonne conductrice, toutes les autres caractéristiques étant égales.

Remarque. - Nous n'avons pas pris en compte les variations des propriétés thermiques $(L, C, K)$ du matériau avec la température :

a) d'une part, dans le domaine de température mentionnée dans la littérature, ces variations ne dépassent pas un facteur 2 et leur influence serait négligeable à la précision de nos calculs ;

b) d'autre part, R. E. Harrington [13] a montré que pour des éclairements intenses la couche superficielle peut être chauffée plus fortement et plus rapidement que ne le prévoit la théorie de la conduction.

b) Influence du pouvoir réflecteur. - Si $R$ désigne le pouvoir réflecteur de la surface, l'énergie absorbée $W_{\mathrm{a}}=W(1-R), W$ étant dans ce cas l'énergie contenue dans le faisceau lumineux. Les valeurs numériques de $R$ données par la littérature sont très grandes, si bien que la majeure partie du rayonnement incident devrait être réfléchie, d'autant plus que l'incidence est oblique.

Pour des valeurs de l'éclairement inférieures au seuil d'apparition des dégâts, la température de la surface devrait être assez bien décrite par :

$\theta_{\mathrm{c}}^{0}=\frac{2}{\sqrt{\pi}} \frac{W(1-R)}{S \sqrt{\tau}} \frac{1}{\sqrt{K C}}=\frac{2}{\sqrt{\pi}} I \tau^{1 / 2}(1-R) \cdot \frac{1}{\sqrt{K C}}$

Ex.: pour le cuivre: $\sqrt{K C}=3,62 \quad 1-R=0,14$ et si $I=10^{+6}$

il vient :

$\begin{array}{lr} & \theta=1,47 \times 10^{+5} \times \tau^{1 / 2} \\ \text { si } \tau=10^{-6} \mathrm{~s} & \theta \simeq 147^{\circ} \mathrm{C} \\ \text { si } \tau=10^{-7} \mathrm{~s} & \theta \simeq 465^{\circ} \mathrm{C}\end{array}$

en supposant une répartition homogène de l'éclairement.

Nous avons vu que la répartition de l'éclairement au foyer de la lentille est loin d'être homogène, de plus des défauts physico-chimiques localisés sur la surface peuvent présenter une plus grande absorption au faisceau lumineux ; tout ceci, combiné à l'inhomogénéité temporelle de l'éclairement entraîne des élévations de température plus importantes sur la surface (apparition des teintes d'échauffement) provoquant l'altération du pouvoir réflecteur.

Pour des éclairements plus élevés, la température de fusion de la couche superficielle est atteinte très rapidement en quelques nanosecondes pour $I=10^{9} \mathrm{~W} / \mathrm{cm}^{2}$ [12]. L'échauffement et la fusion de la surface altèrent grandement son pouvoir réflecteur et l'énergie incidente est d'autant mieux absorbée que le cratère naissant constitue un piège pour la radiation.

Dans ce cas, la température superficielle devrait être assez bien décrite par : 


$$
\theta=\frac{2}{\sqrt{\pi}} \times \frac{I \tau^{1 / 2}}{S \sqrt{\tau}} \times \frac{1}{\sqrt{K C}} .
$$

Ex. pour le cuivre,

$$
\begin{aligned}
\text { si } I & =10^{8} \mathrm{~W} / \mathrm{cm}^{2} & & \theta=6 \times 10^{7} \times \tau^{1 / 2} \\
\tau & =10^{-6} \mathrm{~s} & & \theta=6 \times 10^{4}{ }^{\circ} \mathrm{C} \\
\tau & =10^{-7} \mathrm{~s} & & \theta=1,9 \times 10^{5} \mathrm{C}
\end{aligned}
$$

et pour une répartition homogène de l'éclairement et sans tenir compte de la vaporisation.

Etant donné l'existence d'un maximum d'éclairement sur la zone centrale, il doit y avoir des températures instantanées, localisées, intenses induisant des pressions énormes au sein du liquide en fusion; ce sont ces zones de hautes pressions qui doivent être responsables du jaillissement (hors de la zone focale) de matériau à l'état liquide ou vapeur, matériau qui par refroidissement forme les aigrettes autour du cratère.

Remarques. - 1. En supposant que le pouvoir réflecteur n'est pas altéré pendant la température l'irradiation minimale obtenue est :

$$
\begin{aligned}
\theta_{\operatorname{mini}}=\theta_{\max }(1-\mathrm{R}) & =\theta_{\max } \times 0,14 \\
& \simeq 8,4 \times 10^{3} \mathrm{o} \text { pour } \tau=10^{-6} \mathrm{~s} \\
& \simeq 2,8 \times 10^{4} \mathrm{~K} \text { pour } \tau=10^{-7} \mathrm{~s}
\end{aligned}
$$

ce qui est encore largement suffisant pour provoquer la fusion et la vaporisation de la surface, bien que la vaporisation doive réduire la température superficielle atteinte et provoquer l'absorption d'une partie du rayonnement incident.

2. Les études de A. M. Bonch Bruevich [14] et N. G. Basov [15] ont montré qu'en fait le pouvoir réflecteur des surfaces solides est altéré par l'impact laser à partir d'une valeur de l'éclairement voisine de $10^{8} \mathrm{~W} / \mathrm{cm}^{2}$ et que cette altération est liée à l'échauffement de la surface jusqu'à son point de fusion.

5. VAPORISATION. - a) Conditions de vaporisation. - L'énergie lumineuse contenue dans le faisceau laser incident est absorbée sur la surface $S \simeq 10^{-3} \mathrm{~cm}^{2}$ dans une zone d'épaisseur égale à trois ou quatre fois $z_{0}=\lambda / 2 \pi n_{\mathrm{e}}, z_{0} \simeq 10^{-5} \mathrm{~cm}$. Soit dans un volume $V=S_{\mathrm{z}}=10^{-5} \times 10^{-3}=10^{-8} \mathrm{~cm}^{3}$ de masse $m=\mathrm{V} \rho \simeq 10^{-7} \mathrm{~g}$; comprenant :

$$
n=\frac{6,02 \times 10^{23} \times 10^{-7}}{63}=10^{14} \text { à } 10^{15} \text { atomes }
$$

Dès que la température superficielle atteint la température de vaporisation correspondant à la pression régnant dans l'enceinte (tableau IV), le flux lumineux incident est tel que

$$
(1-R) I \geqslant \frac{T_{V}^{0} \times \sqrt{\pi} \times \sqrt{K C}}{2 \tau^{1 / 2}} .
$$

$3 \times 10^{6}<I<2,1 \times 10^{7}$ si $R$ varie de 0,14 à 0 (cuivre), et en prenant $\tau=10^{-6} \mathrm{~s}$, il va y avoir vaporisation de cette première couche à condition que l'énergie lumineuse contenue dans le faisceau soit suffisante.

L'énergie nécessaire à la vaporisation de cette première couche superficielle de masse $m$ et

$$
W_{m}=m C\left(\theta_{\mathrm{v}}-\theta_{\mathrm{i}}\right)+m\left(L_{\mathrm{f}}+L_{\mathrm{v}}\right)
$$

$\theta_{\mathrm{i}}$ et $\theta_{\mathrm{v}}$ étant la température initiale et la température de vaporisation. $C$ la chaleur spécifique du matériau à l'état solide. Dans le cas du cuivre :

$$
\begin{aligned}
& \begin{aligned}
W_{m} & =m\left(L_{\mathrm{f}}+L_{\mathrm{v}}+C\left(\theta_{\mathrm{v}}-\theta_{\mathrm{i}}\right)\right) \\
& =\left(59 \mathrm{~m}+1270+\left(0,08 \times 10^{3}\right)\right) \\
W_{m} & =1400 \mathrm{cal} / \mathrm{g}
\end{aligned} \\
& \text { soit } \\
& \begin{aligned}
W_{m} & =1400 \times 4,18 \times 10^{-7} \\
& =1,4 \times 4,18 \times 10^{-4} \\
& \simeq 6 \times 10^{-4} \mathrm{~J} \simeq 1 \mathrm{~mJ}
\end{aligned}
\end{aligned}
$$

Dès que cette énergie aura été fournie, la vaporisation va commencer et si l'impulsion lumineuse est suffisamment longue, la surface de la cible va évoluer avec formation d'un cratère.

Cette première couche est la plus longue et la plus difficile à chauffer car elle est initialement très réfléchissante et froide ; après sa vaporisation, étant donné la chute du pouvoir réflecteur, l'absorption sera plus efficace et de plus les couches sous-jacentes seront déjà chauffées par conductibilité thermique.

b) Vitesse de pénétration de la chaleur et vitesse de vaporisation. - 1. Itesse de pénétration de la chaleur. $-z_{\mathrm{e}}=2(k \tau)^{1 / 2}$ étant la profondeur de pénétration de la chaleur dans le matériau pendant la durée de l'impulsion lumineuse $\tau$, la vitesse de pénétration de la chaleur $v_{\mathrm{e}}=z_{\mathrm{e}} / \tau=2(k / \tau)^{1 / 2}$, or $10^{-1}<k<1 \mathrm{~cm}^{2} / \mathrm{s}, 10^{-7}<\tau<10^{-6} \mathrm{~s}$, donc $6 \times 10^{2} \mathrm{~cm} / \mathrm{s}<v_{\mathrm{e}}<6 \times 10^{3} \mathrm{~cm} / \mathrm{s}$.

2. Vitesse $d u$ front de vaporisation $s .-\mathrm{Si} I(t)$ représente le flux d'énergie lumineuse (en $\mathrm{W} / \mathrm{cm}^{2}$ ) absorbé par la surface à l'instant $t, \rho$ la masse spécifique du matériau, c sa chaleur spécifique et $T_{v}$ la température de vaporisation dans les conditions expérimentales, le principe de la conservation de l'énergie entraîne :

$$
s_{(t)}=\frac{I(t)}{\rho\left(L+\mathrm{c} T_{v}\right)}
$$

$\rho\left(L+\mathrm{c} T_{v}\right)$ étant exprimé en $\mathrm{J} / \mathrm{cm}^{3}$, $s_{(t)} \quad$ étant exprimé en $\mathrm{cm} / \mathrm{s}$.

En supposant l'érosion du cratère achevée à la fin de l'impulsion lumineuse, nous pouvons déterminer expèrimentalement la valeur maximale de $s$ en effectuant le rapport $Z_{\mathrm{c}} / \tau$, on trouve

$$
0<s<10^{3} \mathrm{~cm} \times s^{-1} \text {. }
$$


3. Différents régimes de vaporisation. - Suivant les valeurs relatives de $s$ et de $v$, il nous faut envisager trois cas :

a) $s<v$. - La chaleur pénètre rapidement dans la cible mais elle n'est pas suffisante pour vaporiser les couches atteintes. Il y a peu de vaporisation et l'énergie absorbée est perdue par conduction au sein de la cible.

La condition $s<v$ s'écrit :

- pour un éclairement donné $I$

$$
\tau^{1 / 2}<\frac{2 \times \rho\left(L+\mathrm{c} T_{v}\right)}{I(t)} k^{1 / 2}
$$

- pour une durée d'impulsion donnée $\tau$

$$
I_{(t)}<2 \times \rho\left(L+\mathrm{c} T_{v}\right)\left(\frac{k}{\tau}\right)^{1 / 2} .
$$

Par exemple, pour une cible de $\mathrm{Cu}$

$$
\rho\left(L+\mathrm{c} T_{v}\right)=5,25 \times 10^{4} \mathrm{~J} / \mathrm{cm}^{3}
$$

pour $\tau \simeq 10^{-6} \mathrm{~s} \quad I_{t}<1,1 \times 10^{8} \mathrm{~W} / \mathrm{cm}^{2}$

et pour $\tau \simeq 10^{-7} \mathrm{~s} \quad I_{t}<3,6 \times 10^{8} \mathrm{~W} / \mathrm{cm}^{2}$.

$\beta) s \simeq v$. - En même temps que la chaleur pénètre dans la cible, elle la vaporise : c'est la condition pour laquelle le rendement de vaporisation $\rho_{v}$ est maximum.

Dans ce cas, l'épaisseur de matière vaporisée devrait être :

$$
\begin{aligned}
z=\int_{0}^{t} s(t) \mathrm{d} t=\frac{1}{\rho\left(L+\mathrm{c} T_{v}\right)} \times \\
\times \int_{0}^{t} I_{(t)} \mathrm{d} t=\frac{W_{\mathrm{a}}}{\rho\left(L+\mathrm{c} T_{v}\right)} .
\end{aligned}
$$

$W_{\mathrm{a}}$ étant l'énergie lumineuse absorbée par la surface en $\mathrm{J} / \mathrm{cm}^{2}$.

Dans le domaine d'éclairement où $z_{\mathrm{c}}$ croît avec $I$, $10^{6}<I<10^{8} \mathrm{~W} / \mathrm{cm}^{2}$, il doit y avoir une proportionnalité directe entre l'énergie laser et la profondeur atteinte. Expérimentalement nous trouvons

$$
Z_{\mathrm{c}}=f(W)^{0,6},
$$

l'écart par rapport à la linéarité doit provenir des pertes d'énergie par réflexion et par conduction sur la cible, ainsi que par l'absorption d'énergie lumineuse dans la vapeur au voisinage immédiat de la cible.

La condition $s=v$ se traduit par :

$$
\frac{I_{(t)}}{\rho\left(L+\mathrm{c} T_{v}\right)}=2\left(\frac{k}{t_{1}}\right)^{1 / 2}
$$

et $t_{1}$ représente la durée pendant laquelle l'énergie fournie au matériau est entièrement dissipée dans le front de vaporisation, c'est-à-dire le temps au bout duquel la vaporisation pourra se produire si

$$
\begin{aligned}
& I=10^{5} \mathrm{~W} / \mathrm{cm}^{2} \rightarrow t_{1}=1,2 \mathrm{~s} \\
& I=10^{6} \mathrm{~W} / \mathrm{cm}^{2} \rightarrow t_{1}=1,2 \times 10^{-2} \mathrm{~s} \\
& I=10^{7} \mathrm{~W} / \mathrm{cm}^{2} \rightarrow t_{1}=1,2 \times 10^{-4} \mathrm{~s} \\
& I=10^{8} \mathrm{~W} / \mathrm{cm}^{2} \rightarrow t_{1}=1,2 \times 10^{-6} \mathrm{~s} .
\end{aligned}
$$

C'est donc à partir de $I \geqslant 9 \times 10^{7} \mathrm{~W} / \mathrm{cm}^{2}$ que nous trouvons des valeurs de $t_{1}$ compatibles avec la durée de l'émission de notre laser $0,2<\tau<1,5 \mu$ s.

Remarque. - Considérons un cratère d'une certaine profondeur produit dans l'Al, par un faisceau déterminé ( $W$ et $\tau$ connus). Comment doit-on modifier les paramètres lumineux pour obtenir le même cratère dans l'acier ?

Le produit $\rho\left(L+\mathrm{c} T_{v}\right)$ étant supérieur pour l'acier, il faudra plus d'énergie $(W)$ pour vaporiser le même volume. Mais étant donné que la chaleur diffuse moins rapidement, il faut faire agir le faisceau laser plus longtemps pour obtenir la même profondeur. On devra donc augmenter à la fois $W$ et $\tau$.

$\gamma)$ Si $s>v$. - Cas de la saturation.

La condition $s=v$, correspond à une propagation simultanée de la chaleur et du front de vaporisation dans la cible. Si l'énergie lumineuse contenue dans le faisceau devient très supérieure à celle nécessaire pour chauffer et vaporiser l'épaisseur traversée, l'excédent est absorbé par la matière quittant la cible et donne lieu à une vaporisation explosive. $\alpha$ devient à nouveau inférieur à 0,6 .

$s>v$ se traduit par:

$$
\begin{aligned}
& I_{(t)}>\left(\frac{k}{\tau}\right)^{1 / 2}\left[\rho\left(L+\mathrm{c} T_{v}\right)\right] \tau=10^{-6} \mathrm{~s} \\
& I_{t}>6 \times 10^{7} \mathrm{~W} / \mathrm{cm}^{2}
\end{aligned}
$$

et pour

$$
\tau=10^{-7} \quad I_{t}>1,5 \times 10^{8} \mathrm{~W} / \mathrm{cm}^{2} .
$$

En résumé : Pour une valeur déterminée de la durée de l'impulsion lumineuse $\tau$, il existe une valeur de l'éclairement $I_{\text {opt }}$, donc de l'énergie lumineuse, au-delà de laquelle la vaporisation devient importante ; la profondeur du cratère $Z_{\mathrm{c}}$ est reliée à l'énergie lumineuse suivant la loi $Z_{\mathrm{c}}=f(W)^{0,6}$.

Si l'éclairement $I<I_{\text {opt }}$, $\alpha$ devient inférieur à $0,6 \rightarrow$ beaucoup de pertes d'énergie par conduction dans la cible.

Si l'éclairement $I>I_{\text {opt }}$, on a encore $\alpha<0,6 \rightarrow$ beaucoup de pertes d'énergie par absorption dans la vapeur.

c) Quantité de substances vaporisées. - 1. Vaporisation lente $s \leqslant v$ - domaine d'éclairement

$$
10^{6}<I_{m}<10^{8} \mathrm{~W} / \mathrm{cm}^{2} .
$$

Dans le domaine d'éclairement, nous supposons que la vitesse de vaporisation $G$, exprimée en gramme de matière quittant un $\mathrm{cm}^{2}$ de surface pendant une seconde, est donnée par :

$$
G=A\left(P_{s}-P\right)\left[\frac{M}{2 \pi R T}\right]^{1 / 2}
$$

où $A$ est le coefficient de vaporisation, $P_{r}$ la pression de vapeur du matériau à la température, 
$T$ et $P$ la pression partielle du matériau dans l'enceinte.

En supposant cette vitesse uniforme pendant la durée de l'impulsion lumineuse $\tau$, la masse totale de substance vaporisée sur la surface $S$ est :

$m=G S \tau$ et l'énergie nécessaire à cette vaporisation est $W_{m}=m\left(L+\mathrm{c} T_{v}\right)=G S \tau\left(L+\mathrm{c} T_{v}\right)$ et l'éclairement minimal $I_{m}$ nécessaire pour maintenir cette vaporisation est :

$$
I_{m}=\frac{W_{m}}{S \tau}=G\left(L+\mathrm{c} T_{v}\right) .
$$

Dans la zone d'éclairement optimal, $I_{\text {opt }}$, dans laquelle l'énergie fournie par le faisceau laser est la plus efficacement convertie en énergie de vaporisation, on a :

$$
I_{\mathrm{opt}}=2\left(\frac{k}{\tau}\right)^{1 / 2} \rho\left(L+\mathrm{c} T_{v}\right)
$$

D’après (8) et (9), il vient :

$$
G=2\left(\frac{k}{\tau}\right)^{1 / 2} \rho
$$

et la masse totale vaporisée :

$$
m=G S \tau=2(k / \tau)^{1 / 2} \rho S \tau=2(k \tau)^{1 / 2} \rho S .
$$

Ex. : pour le Tantale

$$
\begin{gathered}
k=0,23 \mathrm{~cm}^{2} \mathrm{~s}^{-1} \quad S=10^{-3} \mathrm{~cm}^{2} \quad \rho=16,6 \\
m=1,4 \times 10^{-2} \tau^{1 / 2}
\end{gathered}
$$

soit

$$
6 \times 10^{-6}<m<14 \times 10^{-6} \mathrm{~g}
$$

pour

$$
0,2 \times 10^{-6}<\tau<10^{-6} \mathrm{~s} .
$$

Nous obtenons des valeurs approchées qui nous fixent l'ordre de grandeur $\sim 10^{-6} \mathrm{~g}$, observé expérimentalement, mais notre calcul ne tient pas compte des pertes d'énergie dues à la réflection, à la conduction au niveau de la cible et à l'absorption au niveau de la vapeur, ni de la variation de $k$ avec la température.

D'autre part, $m$ peut aussi s'écrire :

$$
m=\mathrm{GS} \tau=A\left(P_{s}-P\right)(M / 2 \pi R T)^{1 / 2} S \tau
$$

et $m$ dépend beaucoup de la différence $P_{s}-P$.

Quand la cible est placée sous la pression atmosphérique, la vapeur formée est freinée au cours de son expansion par les molécules gazeuses de l'atmosphère au contact desquelles elle se refroidit. Au voisinage immédiat de la cible, la pression partielle du matériau devient rapidement voisine de $P_{s}$ et $G \rightarrow 0$ et le liquide chauffé s'écoule hors de la zone focale.

Par contre, quand la cible est placée dans le vide, la vapeur formée se détend immédiatement, la pression partielle $P$ entourant la vapeur est toujours très petite par rapport à $P_{s}, P \ll P_{s}$, et la vaporisation se pro- longe aussi longtemps que la température superficielle de la cible est telle que la pression de vapeur correspondante est supérieure à la pression régnant dans l'enceinte.

Ceci est mis en évidence dans la figure 9 ; dans le premier cas (sous $P$ atmosphérique), on observe des projections de liquide, figées sur place, le cratère étant très peu profond $\left(z_{\mathrm{c}}<1 \mu\right)$. Dans le deuxième cas (sous vide), la surface interne du cratère est lisse, le matériau absent ayant été vaporisé et la profondeur plus grande $\left(z_{\mathrm{c}} \sim 5 \mu\right)$.

Dans ce dernier cas,

$$
\begin{array}{r}
m \simeq A P_{s}\left(\frac{M}{2 \pi R T}\right)^{1 / 2} S \tau=m=A \frac{P_{s}}{T^{1 / 2}} \cdot\left(\frac{M}{2 \pi R}\right)^{1 / 2} S \tau \\
\frac{P_{s}}{T^{1 / 2}}=\frac{m}{A S \tau}\left(\frac{2 \pi R}{M}\right)^{1 / 2}
\end{array}
$$

la connaissance de ce dernier rapport nous permet d'en déduire la température minimale $T$ atteinte par la surface de la cible.

Ex. : Cible de Tantale $m=10^{-6} \mathrm{~g}, S=10^{-3} \mathrm{~cm}^{2}$

$$
\begin{gathered}
\tau=10^{-6} s-k \simeq 1-M=18 \\
R=10^{8} \text { c.g.s. }, \frac{P_{s}}{T^{1 / 2}}=1,87 \times 10^{6},
\end{gathered}
$$

alors que ce rapport est égal à $1,75 \times 10^{4}$ pour $P=1$ At et $T=5580^{\circ} \mathrm{K}$. La vapeur produite est donc surchauffée (environ $8000^{\circ} \mathrm{K}$ ) et en supposant qu'elle obéit à la loi des gaz parfaits :

$$
P_{s} V=n R T, P_{s}=n \frac{R T}{V}=\frac{G}{M} \frac{R T}{V}
$$

or, d'après (10)

$G=2\left(\frac{k}{\tau}\right)^{1 / 2} \rho=2\left(\frac{k}{\tau}\right)^{1 / 2} \frac{M}{V}=A \times \frac{G}{M} \frac{R T}{V}\left(\frac{M}{2 \pi R T}\right)^{1 / 2}$

d'où

$$
G=2\left(\frac{k}{\tau}\right)^{1 / 2} \cdot \frac{M^{3 / 2}}{\mathrm{AR}^{1 / 2}}\left(\frac{2 \pi}{T}\right)^{1 / 2}
$$

et

$$
m=G S \tau=2(k \tau)^{1 / 2} \cdot S \cdot \frac{M^{3 / 2}}{T^{1 / 2}} \cdot \frac{(2 \pi)^{1 / 2}}{\mathrm{AR}^{1 / 2}}
$$

La masse totale vaporisée croît avec la surface de la cible soumise au rayonnement, la diffusivité thermique $k$, la durée de l'impulsion lumineuse $\tau$, la masse atomique de la cible $M$, et elle décroît avec la température superficielle.

2. Vaporisation explosive $s>v-$ Domaine d'éclairement $I>10^{8} \mathrm{~W} / \mathrm{cm}^{2}$.

Dans ce cas, la température atteinte par la surface $\theta_{s}$ dépasse largement la température de vaporisation correspondant à la pression ambiante.

A ce moment, nous devons avoir sur la surface de 
la cible différentes zones correspondantes aux différences phases du matériau (Fig. 11).

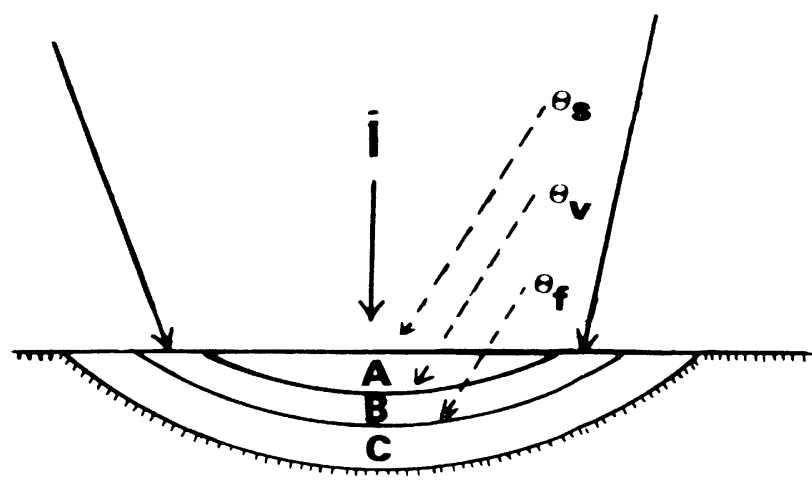

Fig. 11. - Profil des différentes phases sur la surface de la cible.

A correspond à une phase liquide ou gazeuse surchauffée, $\mathrm{B}$ à une phase liquide ordinaire et $\mathrm{C}$ à la phase solide.

Les frontières entre ces différentes phases sont déterminées par la profondeur de pénétration des isothermes de fusion $\theta_{f}$ et de vaporisation $\theta_{v}$.

La phase A surchauffée, sous l'effet d'une variation brutale du gradient de température $\partial \theta / \partial_{z}$, due par exemple à une variation brutale de l'éclairement sur la surface, se vaporise d'une façon explosive.

L'effet de recul dû au départ de cette vapeur provoque l'expulsion du liquide hors de la zone focale, qui par refroidissement forme les aigrettes.

Si $I$ représente le flux de chaleur par unité de surface d'après la théorie de Fourier $I=-K\left(\partial \theta / \partial_{z}\right)$, en négligeant la variation de $K$ avec la température $\theta$, nous pouvons évaluer l'épaisseur des différentes couches A et $\mathbf{B}$.

$$
\begin{gathered}
\frac{\partial \theta}{\partial_{z}}=-\frac{I}{K} \simeq 10^{8}{ }^{\circ} \mathrm{K} \mathrm{cm}{ }^{-1} \\
\int \mathrm{d} z=\int_{T_{1}}^{T_{2}} \frac{K}{I} \mathrm{~d} \theta
\end{gathered}
$$

il vient :

$$
z_{\mathrm{A}}=\frac{K}{I}\left(\theta_{s}-\theta_{v}\right) \quad \text { et } \quad z_{\mathrm{B}}=\frac{K}{I}\left(\theta_{v}-\theta_{f}\right) .
$$

Par exemple pour le cuivre :

$$
\begin{array}{r}
K=3,88 \theta_{v}-\theta_{f} \simeq 2 \times 10^{3}{ }^{\circ} \mathrm{K} \\
I \simeq 2 \times 10^{8} \mathrm{~W} / \mathrm{cm}^{2}
\end{array}
$$

il vient :

$$
z_{\mathrm{B}}=4 \times 10^{-5} \mathrm{~cm} \simeq 0,4 \mu .
$$

Quant à $z_{\mathrm{A}}$, étant donné que

$$
\begin{gathered}
4,3 \times 10^{3}<\theta_{s}<4 \times 10^{4} \mathrm{oK} \\
1 \mu<z_{\mathrm{A}}<8 \mu .
\end{gathered}
$$

La profondeur du cratère $z_{\mathrm{C}}=z_{\mathrm{A}}+z_{\mathrm{B}}$ est de l'ordre de quelques $\mu$, ce qui correspond aux profondeurs effectivement mesurées.
La quantité de substance vaporisée

$$
m=\rho S_{z}=S \frac{K}{I}\left(\theta_{s}-\theta_{v}\right)
$$

étant toujours de l'ordre du $\mu \mathrm{g}$.

L'énergie minimale qu'il faut fournir à cette masse $m$ pour la vaporiser est $W_{n} \simeq m L$; et en supposant que cette énergie est égale à celle qui pénètre la surface ISt, on obtient la durée de temps minimale $t$ au bout duquel cette vaporisation se produit

$$
t=\frac{m L}{I S}=\rho \frac{K L}{I^{2}}\left(\theta_{s}-\theta_{v}\right) .
$$

Si l'impulsion lumineuse est suffisamment longue et si la vapeur émise n'absorbe pas notablement le faisceau lumineux, on peut très bien avoir une série de micro-explosions de ce type ; ce qui expliquerait le fait que $z_{\mathrm{cm}}$ croît avec la durée de l'impulsion lumineuse (Fig. $7 c$ ).

Par exemple, dans le cas du cuivre,

$$
m \simeq \rho S z_{\mathrm{A}} \simeq 4 \times 10^{-6} \mathrm{~g} t \simeq 0,25 \mu \mathrm{s},
$$

il pourrait $y$ avoir une seule micro-explosion pour l'impulsion lumineuse la plus courte $(0,2 \mu \mathrm{s})$ et au maximum six pour la plus longue $(\tau \simeq 1,5 \mu \mathrm{s})$.

IV. Conclusion. - Nous rappelons brièvement les caractéristiques du modèle utilisé pour interpréter la nature des dégâts et la quantité de matière vaporisée :

- l'échauffement superficiel de la cible sur une épaisseur $z_{\mathrm{e}}=(4 k \tau)^{1 / 2}$, sous l'effet de l'éclairement $I$ est

$$
\Delta \theta=\frac{2}{\sqrt{\pi}} I(1-R) \tau^{1 / 2} \times \frac{1}{\sqrt{K C}} .
$$

- la vitesse de pénétration $\mathrm{du}$ front thermique est : $v=2(k / \tau)^{1 / 2}$,

- la vitesse du front de vaporisation

$$
s_{(t)}=\frac{I(t)}{\rho\left(L / c T_{v}\right)} .
$$

La condition $s=v$ se produit pour un éclairement optimal $I_{\text {opt }}$ tel que :

$$
I_{\mathrm{opt}}(1-R)=2\left(\frac{k}{\tau}\right)^{1 / 2} \rho\left(L+\mathrm{c} T_{v}\right)
$$

correspondant à un transfert d'énergie maximal pour la vaporisation.

Dans ce cas, la masse vaporisée

$$
m \sim(k \tau)^{1 / 2} \cdot S \cdot \frac{M^{3 / 2}}{T^{1 / 2}} .
$$

Si $I<I_{\text {opt }}$, l'énergie absorbée est perdue par conduction dans la cible;

Si $I>I_{\text {opt }}$, l'énergie absorbée sert à augmenter l'énergie du matériau éjectée hors de la zone focale.

Un tel modèle ne prétend pas être un reflet rigoureux de la réalité du fait de la complexité temporelle de l'impulsion lumineuse et des variations des propriétés physiques du matériau avec la température. Néan- 
moins, il nous permet de préciser certains ordres de grandeur, en particulier de prévoir les dégâts auxquels on doit s'attendre pour un matériau $(R-K C,-k)$ et pour une impulsion lumineuse donnée $(W, \tau, I)$; $W$ étant de l'ordre de $100 \mathrm{~mJ}, \tau$ de $10^{-6} \mathrm{~s}$ et $I$ variant de $10^{5}$ à $10^{9} \mathrm{~W} / \mathrm{cm}^{2}$.

Par exemple : à partir de la relation donnant $\Delta \theta$, nous pouvons déterminer le seuil d'apparition des dégâts, en calculant l'éclairement nécessaire pour atteindre la température de fusion de la surface, pour une durée d'impulsion $\tau$ donnée.

Si $\tau=10^{-6} \mathrm{~s}$, on trouve $I_{0}=2,5 \times 10^{6} \mathrm{~W} / \mathrm{cm}^{2}$ pour le cuivre et $I_{0}=4,5 \times 10^{5} \mathrm{~W} / \mathrm{cm}^{2}$ pour le plomb.

A l'aide de cette même relation, nous pouvons déterminer la valeur de l'éclairement $I$, nécessaire pour atteindre la température de vaporisation de la surface.

Si $\tau=10^{-6} \mathrm{~s}, I=6 \times 10^{6} \mathrm{~W} / \mathrm{cm}^{2}$ pour le cuivre et $I=2 \times 10^{6} \mathrm{~W} / \mathrm{cm}^{2}$ pour le plomb.

Toutefois cette vaporisation ne devient importante qu'à partir d'une valeur de l'éclairement, $I_{\text {opt }}$, pour laquelle les vitesses de pénétration de la chaleur et de propagation $\mathrm{du}$ front de vaporisation sont égales :

$$
\begin{aligned}
& I_{\text {opt(Cu) }}=1,1 \times 10^{8} \mathrm{~W} / \mathrm{cm}^{2} \\
& I_{\text {op t(Pb) }}=1,2 \times 10^{7} \mathrm{~W} / \mathrm{cm}^{2}
\end{aligned}
$$

et toujours pour $\tau=10^{-6} \mathrm{~s}$.
Dans le meilleur des cas, correspondant à un apport d'énergie lumineuse juste suffisant pour entraîner la vaporisation de l'épaisseur traversée $Z_{\mathrm{e}}$, le rendement de vaporisation n'est seulement que de $10 \%$.

Ce faible rendement est imputable d'une part aux pertes d'énergie par réflexion sur la cible, au début de l'interaction, et par conduction dans le matériau.

D'autre part, nous avons supposé que la vapeur une fois formée était évacuée hors de la zone focale sans absorption supplémentaire d'énergie. Or le phénomène de saturation observé dans l'augmentation des dimensions des cratères avec l'énergie, aussi bien que leur diminution avec la durée de l'impulsion lumineuse montre qu'une partie non négligeable de cette énergie est absorbée par la vapeur aussi longtemps qu'elle occupe la zone focale (16).

Dans la deuxième partie de ce travail, nous n'étudierons plus seulement l'interaction au niveau de la cible, mais les propriétés de la matière éjectée du cratère avec l'énergie qu'elle a pu absorber.

Nous remercions vivement René Stefani, Chef du groupe «Analyse des solides » du laboratoire de Spectrométrie de masse dirigé par Monsieur Cornu, pour l'intérêt qu'il a porté à ces expériences et pour les nombreuses discussions que nous avons eues ensemble lors de la rédaction de ce travail.

\section{Bibliographie}

[1] Honig (R. E.), Woolston (J. R.), « Laser induced emission of electrons, ions and neutrals from solids surfaces ), Appl. Phys. Let., 1963, 2, 138.

[2] Cornu (A.), Rapport interne C. E. A. 1963, n ${ }^{\circ}$ 6215, A. C./J. G.

[3] Eloy (J.), Dumas (J. L.), « Etude des ions produits par impact laser dans une source de spectrométrie de masse ), revue du G. A. M. S., 1966, 3, 251.

[4] Eloy (J. F.), “ Nouvelles expériences au spectromètre de masse à source laser "), revue du G. A. M. S., 1968, 4, 162.

[5] Eloy (J. F.), " Analyse isotopique de surface par un spectromètre de masse à source laser ), revue du G. A. M. S., 1969, 2, 157.

[6] Dumas (J. L.), " Source d'ions pour spectromètre de masse à laser ), revue du G. A. M.S., 1967, 1, 47.

[7] De Metz (J.), Jego (J. M.), Terneaud (A.), ( Evolution temporelle du faisceau d'un laser déclenché ), C. R. Acad. Sci. Paris, 1969, 268B, 1715.

[8] Levine (L. P.), Ready (J. F.), Bernal (E. G.), " Gaz desorption produced by a giant laser pulse ), J. Appl. Phys., 1967, 38, 331.

[9] Ziman (J. M.), " Principles of the theory of solids ), Cambridge University Press, 1964.
[10] Seitz (F.), Koehler (J. S.), « Displacement of atome during irradiations ), Soils Stade Physics : advances in research and applications, Acad. Press. INC. Publishers, New York, 1956, 2, 305.

[11] Carslaw (H. S.), Jaeger (J. C.), " Conduction of heat in solids ), $2^{\mathrm{e}}$ édition, Oxford, 1959, 75.

[12] Ready (J. F.), « Effect due to absorption of laser radiation ), J. Appl. Phys., 1965, 36, 462.

[13] Harrington (R. E.), "Anomalous surface heating rates ), J. Appl. Phys., 1966, 37, 2029.

[14] Bonch Bruevich (A. M.), Imas (Ya A.), Romanov (G. S.), Libenson (M. N.), MAL'TSEv (L. N.), ( Effect of a powerful laser pulse on the reflecting power of a metal ), Sov. Phys. Techn. Phys., 1968, 13, 640.

[15] Basov (N. G.), Boiko (V. A.), Krokhin (O. N.), Semenov (O. G.), Sklizkov (G. V.), « Reduction of reflection coefficient for intense laser radiation on solid surface ), Sov. Phys. Techn. Phys., 1969, 13, 1581.

[16] Weichel (H.), Avizonis (P. V.), « Expansion rates of the luminous front of a laser produced plasma $)$, Appl. Phys. Let., 1966, 9, 334. 